\title{
Myrip Couples the Capture of Secretory Granules by the Actin-Rich Cell Cortex and Their Attachment to the Plasma Membrane
}

\author{
Sébastien Huet, ${ }^{1,2 *}$ Isabelle Fanget, ${ }^{3 \star}$ Ouardane Jouannot, ${ }^{3}$ Patricia Meireles, ${ }^{3}$ Tim Zeiske, ${ }^{3}$ Nathanaël Larochette, ${ }^{3}$ \\ François Darchen, ${ }^{3 *}$ and Claire Desnos ${ }^{3 *}$ \\ ${ }^{1}$ Centre National de la Recherche Scientifique, UMR 6290, Institut Génétique et Développement de Rennes and 2Faculté de Médecine, Université Rennes 1, \\ Université Européenne de Bretagne, Fédération de recherche Biosit, F-35043 Rennes, France, and ${ }^{3}$ Centre National de la Recherche Scientifique, Université \\ Paris Descartes, Sorbonne Paris Cité, UMR 8192, 75270 Paris cedex 06, France
}

Exocytosis of secretory granules (SGs) requires their delivery to the actin-rich cell cortex followed by their attachment to the plasma membrane (PM). How these reactions are executed and coordinated is still unclear. Myrip, which is also known as Slac-2c, binds to the SG-associated GTPase Rab27 and is thought to promote the delivery of SGs to the PM by recruiting the molecular motor myosin Va. Myrip also interacts with actin and the exocyst complex, suggesting that it may exert multiple roles in the secretory process. By combining total internal reflection fluorescence microscopy, single-particle tracking, a photoconversion-based assay, and mathematical modeling, we show that, in human enterochromaffin cells, Myrip (1) inhibits a class of SG motion characterized by fast and directed movement, suggesting that it facilitates the dissociation of SGs from microtubules; (2) enhances their motion toward the PM and the probability of SG attachment to the PM; and (3) increases the characteristic time of immobilization at the PM, indicating that it is a component of the molecular machinery that tether SGs to the PM. Remarkably, while the first two effects of Myrip depend on its ability to recruit myosin Va on SGs, the third is myosin Va independent but relies on the C-terminal domain of Myrip. We conclude that Myrip couples the retention of SGs in the cell cortex, their transport to the PM, and their attachment to the PM, and thus promotes secretion. These three steps of the secretory process are thus intimately coordinated.

\section{Introduction}

Secretory granules (SGs) store water-soluble hormones and release them by exocytosis. A wealth of mechanistic information has been obtained on membrane fusion, but less is known on the recruitment of SGs at the cell periphery and their attachment to the plasma membrane (PM), which is required for the formation of a pool of ready-to-fuse SGs (Verhage and Sorensen, 2008). SGs are formed at the trans-Golgi network and transported along microtubules to the cell periphery (Rudolf et al., 2001). They cannot be transferred directly from microtubules to the PM, but diffuse within the actin-rich cortex until they find an attachment site or undergo another microtubule-based run (Huet et al., 2006). Tethering organelles to the actin cortex is thought to promote their dissociation from microtubules and their accumula-

\footnotetext{
Received June 1, 2011; revised Dec. 2, 2011; accepted Dec. 23, 2011.

Author contributions: S.H., F.D., and C.D. designed research; I.F., O.J., P.M., T.Z., N.L., and C.D. performed research; S.H., I.F., O.J., P.M., F.D. and C.D. analyzed data; F.D. and C.D. wrote the paper.

This work was supported by the Centre National de la Recherche Scientifique. F.D. was supported by INSERM. We thank Philippe Rostaing and Laure Wingertsmann from the electronic microscopy platform at the Ecole Normale Supérieure, Paris, and Dr. Ana-Maria Lennon-Duménil for her critical reading of this manuscript.

*S.H., I.F., F.D., and C.D. contributed equally to this work.

Correspondence should be addressed to Dr. François Darchen, Université Paris Descartes, Centre National de la Recherche Scientifique, UMR8192, 45 Rue des Saints-Pères, 75270 Paris Cedex 06, France. E-mail: francois.darchen@parisdescartes.fr.

DOI:10.1523/JNEUROSCI.2724-11.2012

Copyright $\odot 2012$ the authors $\quad 0270-6474 / 12 / 322564-14 \$ 15.00 / 0$
}

tion at the cell periphery, and thus secretion (Seabra and Coudrier, 2004; Darchen and Desnos, 2012). However, excessive binding to actin restricts the mobility of SGs and their availability for release (Desnos et al., 2003). Actin dynamics (Malacombe et al., 2006) as well as molecular motors such as myosin Va (MyoVa) are thought to facilitate the motion of SGs through the actin-rich layer.

MyoVa is associated with SGs and its inhibition impairs SG docking and secretion (Rose et al., 2003; Rudolf et al., 2003, 2011; Varadi et al., 2005; Desnos et al., 2007b). MyoVa is recruited on SGs by the GTPase Rab27a and Myrip (Fukuda and Kuroda, 2002; Desnos et al., 2003; Waselle et al., 2003; Imai et al., 2004). Myrip, which is also known as Slac-2c, is expressed on SGs and retinal melanosomes (Darchen and Desnos, 2012), and like MyoVa, Rab27a, and Rab27b, it controls secretion (Desnos et al., 2003; Waselle et al., 2003; Imai et al., 2004; Ivarsson et al., 2005; Mizuno et al., 2011). Myrip interacts with Rab27:GTP via an N-terminal helix, and with myosin VIIa or MyoVa via a central domain (El-Amraoui et al., 2002; Fukuda and Kuroda, 2002; Desnos et al., 2003; Kuroda and Fukuda, 2005; Klomp et al., 2007; Lopes et al., 2007; Ramalho et al., 2009). Interestingly, through its C-terminal region, Myrip also interacts with sec6 and sec8, two components of the exocyst complex, and with actin (ElAmraoui et al., 2002; Fukuda and Kuroda, 2002; Desnos et al., 2003; Goehring et al., 2007), suggesting that Myrip can exert MyoVa-independent functions. 
Here, we measured the dynamics of SGs beneath the PM in neuroendocrine cells using total internal reflection fluorescence microscopy (TIRFM) combined with single-particle tracking and incorporated the data into a mathematical compartment model of SG trafficking. We found that Myrip plays a dual role in SG dynamics. First, Myrip recruits MyoVa and promotes the retention of SGs in the actin-rich cortex and their transport toward the PM. Second, it allows their docking at the PM. By coupling the delivery of SGs to the PM and the docking reaction, Myrip thus plays a key role in the secretory process.

\section{Materials and Methods \\ Materials}

Anti-chromogranin A/B antibodies were from Abcam; goat anti-Myrip antibodies were from Everest Biotechnology. The anti-myosin Va (Espreafico et al., 1992) was a gift from R. E. Cheney (University of North Carolina, Chapel Hill, NC) or obtained from Sigma (LF-18); anti-actin (AC-74) and anti tubulin (TUB 2.1) antibodies were from Sigma, and anti-Myc 9B11 was from Cell Signaling Technology. Cell culture reagents were obtained from PAA Laboratories. DNA purifications were done using kits from Macherey-Nagel. Enzymes for molecular biology were from New England Biolabs.

\section{Constructs}

To generate a plasmid encoding Dendra2-tagged neuropeptide-Y (NPY), p-Dendra2C (Evrogen) was digesting using BamHI/EcoRI and subcloned into pCDNA.3.1. The resultant plasmid was cut with BamHI/ NotI, and the fragment was used to replace the BamHI-NotI fragment in pNPY-GFP to generate an in-frame fusion between NPY and Dendra2. The sequence of inserts was confirmed by automated DNA sequencing. pcDNA3-mycRab1lb was a gift from M. Cormont (INSERM U895, Centre Méditerranéen de Médecine Moléculaire, Nice, France). Vectors encoding human Myc-tagged-Myrip constructs, human myosin Va, and NPY constructs have been described previously (El-Amraoui et al., 2002; Desnos et al., 2007b). To generate a Myrip rescue construct, three silent mutations corresponding to nucleotides 2, 9 and 11 of Myrip-siRNA1 (sense strand) were introduced by PCR-based mutagenesis in the Myctagged Myrip construct. We verified by immunofluorescence that the expression of this rescue construct was not reduced by Myrip-siRNA1, in contrast to that of the original Myrip construct.

\section{Cell culture, transfection, and silencing}

The BON cell line has been established from a lymph nod metastasis of a human pancreatic carcinoid tumor (Evers et al., 1991) and were provided by C. M. Townsend (University of Texas, Medical Branch, Galveston, TX). In culture, a large majority of the cells extend several processes enriched in SGs. Cells were cultured in Ham's F-12/DMEM with 10\% FBS at $37^{\circ} \mathrm{C}$ under $5 \% \mathrm{CO}_{2}$. Cells were plated onto collagen-coated glass coverslips before transfection. TIRFM observations were made on collagen-coated glass-bottom dishes (MatTEK or World Precision Instruments). SiRNA duplexes were provided by MWG Biotech. We used three already validated siRNAs directed against human MyoVa (MyoVasiRNA1, 5' -GAACAAAUGUGCACUCUUUUU; MyoVa-siRNA2, 5' -AA AGUAAGGUCGUUGCUAAUU; MyoVa-siRNA3, 5'-AACUGACUAC CUGAAUGAUGA) (Desnos et al., 2007b; Lindsay and McCaffrey, 2011) and two siRNAs targeting Myrip (Myrip-siRNA1, 5'-UAAGUGAGCU GAAGCAGAAUU; Myrip-siRNA2, 5' - UGGCAGUGAUUCAACAUU $\mathrm{U})$. The control siRNAs targeting EGFP or luciferase were from Eurofin MWG Operon. Transfection of siRNAs (30 nM) was done with interferin (Polyplus Transfection). Twenty-four hours later, cells were transfected with plasmids using lipofectamine 2000 (Invitrogen). In some cases, BON cells were transfected by electroporation in the presence of siRNA $(120 \mathrm{nM})$ and $24 \mathrm{~h}$ later with siRNAs and interferin. In brief, $1.5 \times 10^{6}$ cells were collected in $50 \mu \mathrm{l}$ of PBS containing vector DNAs $(2-5 \mu \mathrm{g})$, electroporated $(600 \mathrm{~V} / \mathrm{cm}, 8 \times 3 \mathrm{~ms})$ using a PS10 electropulsator (Jouan), and recovered in warm culture medium before plating. Experiments were done $72 \mathrm{~h}$ after initiation of RNA interference. Cell extracts were prepared in parallel for Western blot analysis for protein expression. The blots were scanned and quantified using Image J software (rsbweb. nih.gov/ij/). Levels of actin or tubulin were used to normalize the results from different samples.

\section{Coimmunoprecipitation}

BON cells were transfected $2 \mathrm{~d}$ before the experiment with vectors encoding myc-tagged Myrip and GFP-MyoVa tail. Immunoprecipitation was performed as described previously (Desnos et al., 2003) on cell homogenates prepared in $1 \%$ Triton X-100 in the presence of $2 \mu \mathrm{M}$ latrunculin B (Calbiochem) to recover Myrip that otherwise bound strongly to actin filaments. Extracts were incubated with protein G-Sepharose beads (GE Healthcare) conjugated with required antibodies at $4^{\circ} \mathrm{C}$ for $2 \mathrm{~h}$. After extensive washes and elution in Laemmli sample buffer, eluates were analyzed by SDS-PAGE and immunoblotting.

\section{Immunofluorescence microscopy}

Immunocytochemistry was performed as described previously (Desnos et al., 2003). Secondary antibodies were coupled to Cy-3 (Jackson ImmunoResearch Laboratories), Alexa-488, Alexa-568, or Alexa-350 (Invitrogen). Cells were visualized using a $z$-motorized Nikon inverted microscope TE2000E equipped with a $100 \times$ objective (NA, 1.4) and a CoolSnap ES CCD camera (Roper Scientific). When indicated, $Z$-series were acquired with $\Delta z=200 \mathrm{~nm}$, and image stacks were restored using the MetaMorph point spread function-based deconvolution software (Molecular Devices). To evaluate the association of MyoVa with SGs, cells were imaged using the same laser and camera settings. We first identify SGs as NPY-monomeric red fluorescent protein (mRFP)-labeled structures whose brightest pixel had a fluorescence intensity $>1.25$ times that of the background fluorescence measured in several areas of the cell. Then, we categorized a SG as MyoVa positive if the green fluorescence of this brightest pixel was $>1.25$ times that of the background green fluorescence of this cell.

\section{High-pressure freezing immunoelectron microscopy}

BON cells were cultured on thermanox coverslips (Nunc) and fitted in a $6 \mathrm{~mm}$ specimen carrier for rapid freezing in a Leica HPM 100 machine under high pressure (2100 bars). Samples were then rapidly transferred to liquid nitrogen for storage. Cryosubstitution and embedding were performed in a Reichert AFS apparatus (Leica), first in acetone with $0.1 \%$ tannic acid at $-90^{\circ} \mathrm{C}$ for $40 \mathrm{~h}$ with one change of solution, and then in acetone with $2 \%$ osmium during the last $7 \mathrm{~h}$. The cultures were slowly $\left(5^{\circ} \mathrm{C} / \mathrm{h}\right)$ warmed to $-20^{\circ} \mathrm{C}$ and incubated for additional hours before being warmed $\left(10^{\circ} \mathrm{C} / \mathrm{h}\right)$ to $4^{\circ} \mathrm{C}$. After several rinses with acetone, the samples were warmed to room temperature and incubated in 50\% acetone $/ 50 \%$ Araldite (Polysciences) for $1 \mathrm{~h}$, followed by $10 \%$ acetone $/ 90 \%$ Araldite for $2 \mathrm{~h}$. They were then incubated twice in pure Araldite for $2 \mathrm{~h}$. Coverslips were mounted on resin block and cut in parallel to the plan of cells after removing the thermanox coverlip. Sections ( $80 \mathrm{~nm}$ thick) were cut using a Leica Ultracut $\mathrm{E}$, counterstained by incubation with $2 \%$ uranyl in water for $10 \mathrm{~min}$ and lead citrate for $10 \mathrm{~min}$. The sections were observed in a Philips TENAI 12 (FEI).

\section{TIRFM}

TIRFM setup. BON cells were transferred into Locke's solution containing the following (in mM): 5.6 glucose, $3.6 \mathrm{HCO}_{3}^{-}, 159.6 \mathrm{Cl}^{-}, 157.6 \mathrm{Na}^{+}$, $5.6 \mathrm{~K}^{+}, 5$ HEPES-NaOH, $2.5 \mathrm{CaCl}_{2}, 1.2 \mathrm{MgCl}_{2}$. TIRFM imaging was performed on a custom setup described previously (Huet et al., 2006). The penetration depth $\delta$ (the distance along the $z$-axis over which fluorescence declines $e$-fold) of the evanescent field used to excite the fluorophore was set to $150 \mathrm{~nm}$. Under the conditions of observation used, one pixel corresponded to $107.5 \mathrm{~nm}$. Stream acquisitions were performed at $9.98 \mathrm{~Hz}$ for $40-60 \mathrm{~s}$ with an exposure time of $100 \mathrm{~ms}$. Selective excitation was obtained using argon laser lines at 488 and $514 \mathrm{~nm}$, and optical filters (bandpass, 500-540 nm; high-pass, >565 nm) were used for the emission (Melles Griot).

Image analysis. The density of fluorescent structures was evaluated using Multidimensional Image Analysis (MIA) software (a segmentation algorithm based on wavelets) (Racine et al., 2006) and expressed as the number of NPY-positive structures observed in the evanescent field divided by the size of the cell footprint. 
From stacks of images, two-dimensional $(x-y)$ trajectories were obtained by single-particle tracking using MIA software. Mean square displacement (MSD) in the $x-y$ plane was computed using custom routines, and the diffusion coefficient $D_{x y}$ was then calculated as $D_{x y}=s / 4$, with $s$ being the slope of the linear fit to the first 15 points of the MSD curve. To measure $D_{x y}$ variations along a given trajectory, a rolling analysis window of $2.5 \mathrm{~s}$ was used. For each position of the window, $D_{x y}$ was calculated as described above, and the obtained value was assigned to each point of the trajectory included in this window. Since each point of the trajectory appears in different windows, several $D_{x y}$ values were assigned to each point and finally averaged. Immobilization periods were defined as portions of trajectories during which $D_{x y}$ drops below a threshold value $D_{\min }$ $=5 \times 10^{-4} \mu \mathrm{m}^{2} \cdot \mathrm{s}^{-1}$, i.e., five times the minimal $D_{x y}$ measurable with our experimental setup (estimated from the tracking of fluorescent beads immobilized on the coverslip) (Huet et al., 2006). As random walk trajectories may also feature periods of slow motion, we used the duration of immobilization periods as a further criterion of SG attachment. Survival curves for immobilization times (i.e., the cumulative number of immobilization events per trajectory plotted as a function of their minimum duration) were computed for each cell and averaged over the different cells. The best fit for the curves was obtained with the sum of two exponentials, $N(t)=N_{1} \exp \left(-t / \tau_{1}\right)+N_{2} \exp \left(-t / \tau_{2}\right)$, using SigmaPlot (Systat Software). $N_{1}$ and $N_{2}$ represent the abundance of each component, and $\tau_{1}$ and $\tau_{2}$ their time constants, respectively. The long-lasting component was taken as an index of SG stalling.

Minimizing the cross talk between attachment to the PM and diffusive periods. Based on the two characteristic times observed in survival plots of immobilization times, we calculated a threshold value for the immobilization duration to separate long-lasting immobilization periods from the poorly defined short-lasting immobilization periods. This threshold was calculated to minimize the cross talk between the two populations in the survival curves. The normalized distributions for the short $\left(D_{s}(t)\right)$ and long $\left(D_{1}(t)\right)$ immobilization periods are given by the following equations:

$$
\begin{aligned}
& D_{\mathrm{s}}(t)=\frac{1}{\tau_{\mathrm{s}}} \exp \left(\frac{-t}{\tau_{\mathrm{s}}}\right) ; \\
& D_{1}(t)=\frac{1}{\tau_{1}} \exp \left(\frac{-t}{\tau_{1}}\right) .
\end{aligned}
$$

For a given threshold $t_{\text {thresh }}$, the cross talk between the two distributions is equal to the following:

$$
I\left(t_{\text {thresh }}\right)=\int_{0}^{t_{\text {thresh }}} D_{1}(t) d t+\int_{t_{\text {thresh }}}^{\infty} D_{s}(t) d t .
$$

By calculating the two integrals, we obtain the following:

$$
I\left(t_{\text {thresh }}\right)=1+\exp \left(\frac{-t_{\text {thresh }}}{\tau_{\mathrm{s}}}\right)-\exp \left(\frac{-t_{\text {thresh }}}{\tau_{1}}\right) .
$$

It is thus possible to obtain the threshold duration which minimizes the cross talk between the two populations:

$$
t_{\text {thresh }}=\frac{\tau_{\mathrm{s}} \times \tau_{\mathrm{t}}}{\tau_{1}-\tau_{\mathrm{s}}} * \ln \left(\frac{\tau_{1}}{\tau_{\mathrm{s}}}\right)
$$

Based on this calculation, we obtained threshold durations between 6 and $8.5 \mathrm{~s}$ for the different experiments. Subtrajectories corresponding to directed motion were manually selected; stalled periods include all immobilization periods lasting longer than the threshold value defined above. The remaining subtrajectories were considered as diffusive.

Detection of false positive stalling periods within simulated Brownian trajectories and diffusing fluorescent beads. Three-dimensional random walk trajectories were simulated as described by Huet et al. (2006). The diffusion coefficient $\left(25 \times 10^{-4} \mu \mathrm{m}^{2} \cdot \mathrm{s}^{-1}\right)$, length of the trajectories $(10$ to $20 \mathrm{~s}$ ) and time step between consecutive positions ( $0.1 \mathrm{~s})$ were chosen to match the values obtained for SGs. Stalling events were detected with the approach used for SGs. The survival curves for immobilization times were based on the simulation of 5000 trajectories.

Time-lapse stacks displaying the diffusion of latex fluorescent beads ( $500 \mathrm{~nm}$ in diameter) within a glycerol/water $\operatorname{mix}$ ( $75-80 \%$ of glycerol by volume) were acquired by TIRF microscopy. The beads were tracked using the MIA software. The average diffusion coefficient of these beads $\left(D_{x y} \approx 130 \times 10^{-4} \mu \mathrm{m}^{2} \cdot \mathrm{s}^{-1}\right)$ was approximately five times higher than the value measured for SGs in control conditions. However, to properly estimate the contribution of false positive stalling events within the SG survival curves using the analysis of the bead dynamics, it was necessary to match the ratio between the average diffusion coefficient and the threshold value $D_{\min }$ for the beads and the SGs. Consequently, stalling periods within bead trajectories were detected with a threshold $D_{\min }=$ $25 \times 10^{-4} \mu \mathrm{m}^{2} \cdot \mathrm{s}^{-1}$ and not $5 \times 10^{-4} \mu \mathrm{m}^{2} \cdot \mathrm{s}^{-1}$, as used for the SGs. The survival curves for immobilization times were based on 69 bead trajectories whose duration matches that of SGs $(\sim 16 \mathrm{~s})$.

Estimation of the diffusion anomaly. In case of anomalous subdiffusion, the MSD curves can be fitted by the following:

$$
\mathrm{MSD} \propto D \cdot \Delta t^{\alpha},
$$

with $\alpha \leq 1$. In the logarithmic representation, Equation 1 becomes

$$
\log (\mathrm{MSD}) \propto \alpha * \log (\Delta t) .
$$

Thus, the MSD curves calculated from the SG trajectories were plotted in logarithmic representation and fitted using a linear regression, the slope of the fit corresponding to the anomaly parameter $\alpha$. For the estimation of the diffusion anomaly during stalling and nonstalling periods, the MSD curves were calculated for stretches of trajectories lasting $>5 \mathrm{~s}$. A median MSD was calculated for each cell, and the fit was performed over the first 25 points of each MSD curve.

Measuring peripheral SG retention by photoconversion of NPY-Dendra2. NPY was fused to Dendra2. Dendra2 is a photoactivable fluorescent protein whose emission spectrum shifts from green to red upon brief UV light exposure (Chudakov et al., 2007). A $405 \mathrm{~nm}$ diode laser (50 mW; Laser Components) was coupled to our TIRF microscope and aligned with the argon laser using a dichroic mirror. Two days after transfection with NPY-Dendra2, Myrip siRNA, or Myrip constructs, the cells were observed by TIRFM with a penetration depth of $100 \mathrm{~nm}$. A $2 \mathrm{~s}$ exposure to UV evanescent light was sufficient to photoconvert $64 \pm 8 \%$ ( $n=15$ cells) of the green vesicles present in the TIRFM field. Red signals three times above the background were obtained. Increasing the photoactivation time over $2 \mathrm{~s}$ did not significantly increase the red signal but increased the variability. After photoconversion, images were acquired for $1 \mathrm{~min}$ at $0.5 \mathrm{~Hz}$ using an exposure time of $200 \mathrm{~ms}$. Dendra2 was not significantly photoconverted by such illumination at $514 \mathrm{~nm}$. Images were quantitated using ImageJ. For each cell, the background was measured in areas devoid of SGs and subtracted to all pixel values. Cell boundaries were manually drawn, and the total fluorescence intensity was integrated over the depicted area. Alternatively, images were segmented using MIA software, and the number of vesicles was counted on each frame. Photobleaching was estimated on live cells by measuring the fluorescence decay of immobile SGs, yielding an upper bound estimate of bleaching since immobile SGs are generally attached to the PM and thus close to the interface where the TIRF field is generated. Diffusion of NPY-mRFP within individual SGs may also contribute to the diminution of the fluorescence signal over time. However, this fluorescence loss is likely to be the same under the different conditions since we found, using electron microscopy, that Myrip siRNAs do not change the size of SGs.

Simulation of the photoconversion experiments. To simulate the photoconversion experiments, we built up a kinetic model in which SGs can occupy different states: "C" for SGs localized at the cell center, "A" for SGs in the actin cortex, and "D" for SGs attached to the PM. TIRF microscopy does not allow imaging all the SGs present at the cell cortex but only those which are localized in the vicinity of the glass coverslip. To account for this limitation, we included in the model two peripheral SG populations, only one of them being accessible by TIRF imaging. 
Due to the short evanescent depth used for photoconversion $(\sim 80 \mathrm{~nm}$ at $405 \mathrm{~nm}$ ) most of the photoconverted NPY-Dendra labeled SGs were considered as docked at the PM immediately after the photoconversion. We used this initial condition for starting our simulations. Since the evanescent depth used for imaging is longer than the one used for photoconversion (100 $\mathrm{nm}$ instead of $80 \mathrm{~nm}$ ), we considered that we were imaging all the docked SGs and $25 \%$ of the SGs located in the actin cortex. Because this proportion $p_{\text {act }}$ of SGs located in the actin cortex which can be imaged using a $100 \mathrm{~nm}$ evanescent depth could not be estimated precisely, we performed simulations in which the value of $p_{\text {act }}$ ranged between 0 and $50 \%$. In all cases, we observed a biphasic behavior, the fast phase corresponding to the equilibration between the pool at the $\mathrm{PM}$ and the one in the actin, and the slow phase associated with SG movement toward the cell interior. Varying $p_{\text {act }}$ from 0 and $50 \%$ only led to a moderate change in the relative amplitude of the two phases (data not shown).

The exchange rates between the three states were derived from the analysis of SG dynamics by TIRF microscopy (Table 1). To estimate the rates $k_{1}$ and $k_{1}{ }^{\prime}$ corresponding to the transition $\mathrm{C} \rightarrow \mathrm{A}$ and $\mathrm{C} \rightarrow \mathrm{A}^{\prime}$, with $\mathrm{A}^{\prime}$ being the population of SGs in the actin-rich layer not visible in TIRFM, we had to estimate the relative SG populations in the different compartments at steady state. Based on the images obtained by electron microscopy, we observed that about two-thirds of the SGs display a cortical localization in control cells. Our analysis of SG mobility also showed that, in control conditions, the density of SGs in the actin cortex and at the PM are similar $(A \approx D)$. Finally, we estimated the proportion of SGs imaged by TIRF as compared to the total number of SGs in the cell. On deconvolved wide-field images of NPY-GFP-expressing BON cells, we counted a total number of labeled vesicles of $\sim 1500$ SGs. Knowing that we observed $\sim 150$ SGs by TIRF, we considered that $\sim 10 \%$ of the total number of labeled SGs were visible with this approach. Together, these different results allowed estimating the steady SG populations in the different compartments. We obtained $C \approx 33 \%, A \approx D \approx 8 \%$, and $A^{\prime} \approx$ $D^{\prime} \approx 25.5 \%$. Since $k_{1}=k_{-1} * A / C$ and $k_{1}{ }^{\prime}=k_{-1}{ }^{*} A^{\prime} / C$, at steady state, we could estimate that, in the control cells, $k_{1} \approx k_{-1}{ }^{\star} 0.24$ and $k_{1}{ }^{\prime} \approx$ $k_{-1} * 0.77$.

\section{Statistical analyses}

Values are given as mean \pm SE. The significance of differences between two conditions was calculated with Mann-Whitney $U$ test or Student's $t$ test when data were normally distributed. For multiple comparisons, we used the Kruskal-Wallis test or ANOVA (normal distribution), followed by Dunn's or Tukey's post-tests, respectively, using GraphPad Prism version 5.04.

\section{Results}

\section{Myrip recruits MyoVa on secretory granules}

Myrip forms a complex with MyoVa and Rab27a and is therefore thought to mediate the recruitment of MyoVa onto SGs. To test this possibility, we measured the association of a GFP-tagged construct comprising the globular tail of MyoVa and the exons A, C, E and F (GFP-MyoVa tail) with SGs in an enterochromaffin cell line $(\mathrm{BON})$ derived from a human carcinoid tumor. BON cells store various peptides and serotonin in SGs and secrete them (Kim et al., 2001). They express Rab27a, Myrip, and MyoVa and the three proteins are associated with SGs as in other endocrine cells (Fig. 1) (Desnos et al., 2003, 2007b). Under control conditions, $60 \%$ of the NPY-mRFP labeled SGs were decorated with a GFP-MyoVa tail (Fig. $1 A-C, H$ ). In contrast, in cells treated with a silencing RNA duplex (siRNA) directed to Myrip mRNA (Fig. $1 G)$, the fraction of SGs labeled by GFP-MyoVa tail was reduced to $\sim 20 \%$ (Fig. $1 D-F, H$ ). Moreover, in $\sim 60 \%$ of Myrip-siRNAtreated cells, the GFP-MyoVa tail exhibited a marked soluble cytosolic pattern without any visible enrichment on intracellular structures (Fig. $1 I, J$ ). Such increased cytosolic distribution was observed only in $22 \%$ of control cells. Overall, the intensity of the cytosolic GFP-MyoVa tail in cell regions devoid of SGs was doubled in Myrip-siRNA-treated cells compared to control cells (mean fluorescence intensity, control cells, $288 \pm 32$; Myrip knockdown cells, $595 \pm 58 ; p<0.0001 ; n=30$ cells). The association of MyoVa with SGs was rescued by expressing an siRNAinsensitive Myrip construct, arguing against an off-target effect of Myrip siRNAs (Fig. $1 H$ ). These observations indicate that Myrip is needed for the recruitment of MyoVa on SGs.

\section{The intracellular distribution of SGs depends on Myrip}

Next, we analyzed the effect of Myrip on the intracellular distribution of SGs. In the majority of control cells, SGs accumulate at the cell periphery and in cell extensions (Figs. 1, 2A, left). In contrast, SGs were evenly distributed in the cytoplasm or concentrated near the nucleus (Fig. $2 \mathrm{~A}$, middle, right) in most of the Myrip knockdown cells and in cells in which the Rab-binding domain of Myrip (Myrip-RBD) was expressed to inhibit the binding of endogenous Myrip to Rab27a (Fig. 2B). This effect was first evaluated by visual inspection of cells and manual determination of the fraction of cells exhibiting at least one cell extension with a SG density higher than in the cell center (Fig. 2B). A more quantitative analysis of the mean distance of SGs to the cell center confirmed these results (Fig. 2C). Noteworthy is that the effect of Myrip silencing on SG distribution was rescued by expressing a siRNA-insensitive Myrip construct (Fig. 2B,C). Finally, BON cells were imaged by TIRFM to image NPY-labeled SGs present in the subplasmalemmal region (Huet et al., 2006; Desnos et al., 2007b). Most of the SGs observed by TIRFM are localized within the actin-rich cell cortex or at the PM (Oheim and Stuhmer, 2000; Johns et al., 2001; Huet et al., 2006). Myrip silencing and overexpression of Myrip-RBD significantly diminished the number of SGs present in the evanescent field, compared with control cells (Fig. 2D). In contrast, overexpression of full-length Myrip (Myrip-FL) but not of a C-terminally truncated Myrip (Myrip- $\Delta \mathrm{C}, 1-665$ ) had a tendency to increase SG density in the subplasmalemmal region (Fig. 2D), suggesting that the C-terminal region of Myrip contributes to SG retention, although it is not needed for the interaction with MyoVa (Desnos et al., 2003; Kuroda and Fukuda, 2005). We conclude that Myrip promotes the recruitment or the retention ("capture") of SGs near the PM.

To characterize the effect of Myrip on SG distribution by independent means, we measured the distance of SGs to the PM by electron microscopy. BON cells were treated for $3 \mathrm{~d}$ with control siRNAs or Myrip siRNAs, fixed by high-pressure freezing and embedded after cryosubstitution. This technique was shown to preserve the cell architecture and the position of vesicles with respect to the PM (Siksou et al., 2007). Electron-dense SGs were easily identified on EM pictures (Fig. $3 A$ ), and their distance to the PM was determined. In agreement with TIRFM data, the number of SGs positioned within $300 \mathrm{~nm}$ of the PM was reduced in Myrip knockdown cells, compared with control cells (Fig. 3B). Interestingly, the effect of Myrip silencing on the number of SGs very close to the PM $(<50 \mathrm{~nm})$ or morphologically attached to the PM $(<15 \mathrm{~nm})$ was even more pronounced. Noteworthy, Myrip silencing had no effect on the size of SGs (not shown) or on the total number of SGs (cells treated with control siRNAs, $2.17 \pm 0.27 \mathrm{SG} / \mu \mathrm{m}^{2}, n=32$ cells; Myrip knockdown cells, $2.32 \pm 0.54 \mathrm{SG} / \mu \mathrm{m}^{2}, n=30$ cells).

In agreement with this defect in SG recruitment at the PM, Myrip silencing reduced the secretion of serotonin in BON cells (Fig. 2E), as it does in other neuroendocrine cells (Fukuda and Kuroda, 2002; Desnos et al., 2003; Waselle et al., 2003; Imai et al., 2004; Ivarsson et al., 2005; Mizuno et al., 2011). 


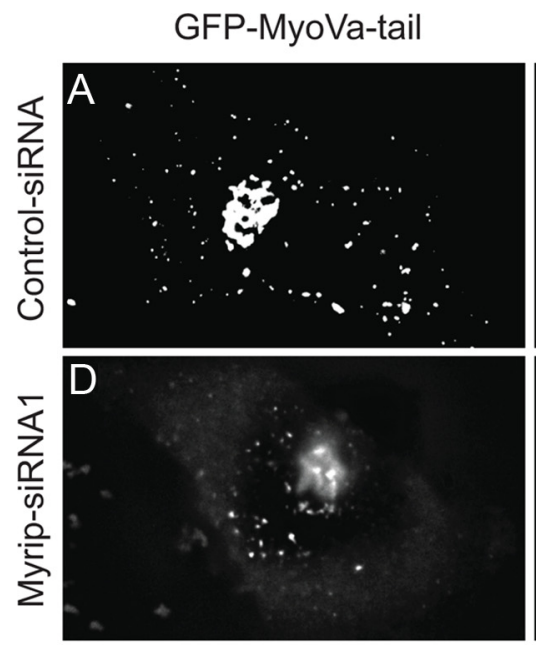

G

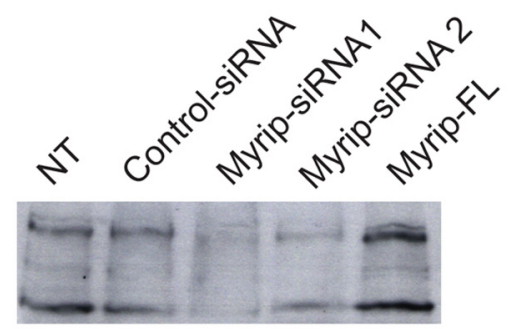

NPY-mRFP
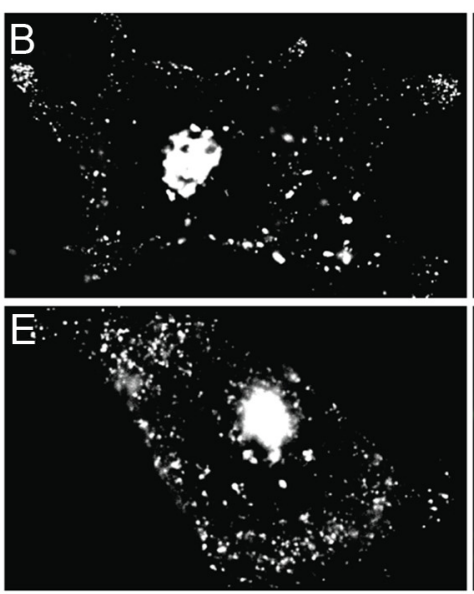

Merge
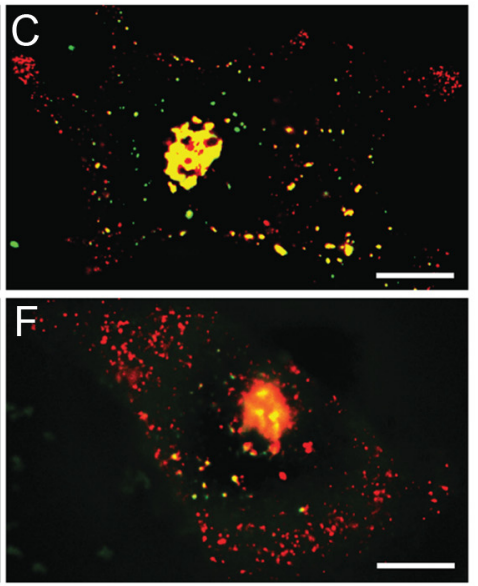

$\mathrm{H}$

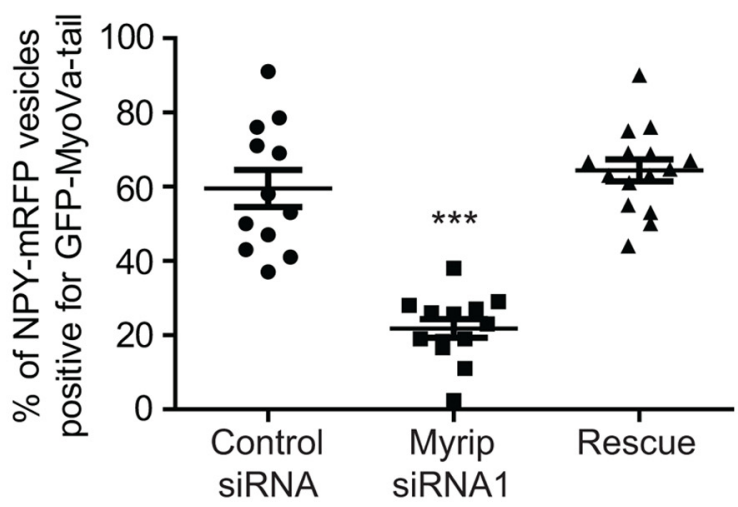

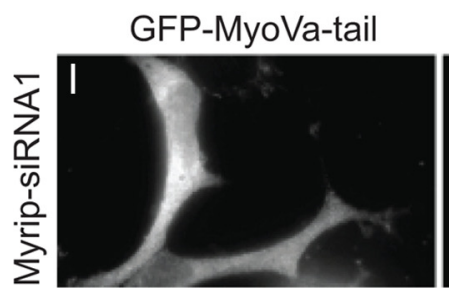
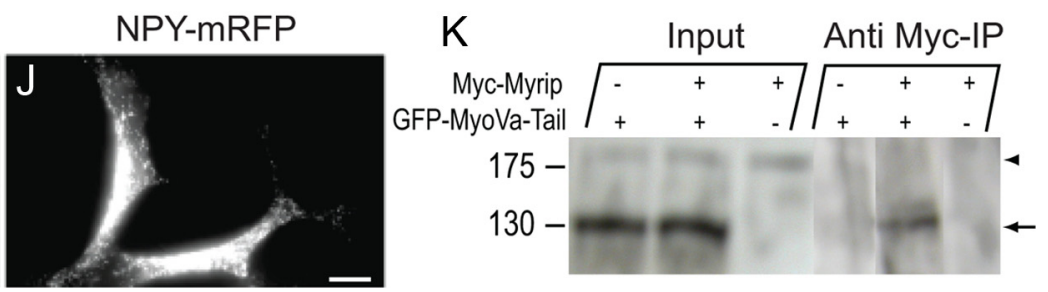

Figure 1. Myrip recruits myosin Va onto SGs. $\boldsymbol{A}-\boldsymbol{F}$, To evaluate the association of MyoVa to SGs, BON cells were successively transfected with NPY-mRFP $(\boldsymbol{B}, \boldsymbol{E})$ and with GFP-MyoVa tail $(\boldsymbol{A}, \boldsymbol{D}) 3 \mathrm{~d}$ and $16 \mathrm{~h}$ before cell fixation, respectively. In addition, cells were transfected with luciferase-targeting siRNAs as a control $(\boldsymbol{A}-\boldsymbol{C})$ or with Myrip siRNA1 (D-F).Z-Zstacks of epifluorescence images were restored by deconvolution. G, Knockdown of Myrip expression. Three days after transfection of siRNAs, Myrip levels were analyzed by immunoblotting in nontransfected (NT) cells, in cells treated with a control siRNA, with siRNAs directed to Myrip mRNA, and in cells overexpressing Myrip-FL. Myrip levels were lowered by $80 \pm 1.8 \%$ (Myrip-siRNA1) or by $63 \pm 1.8 \%$ (Myrip-siRNA2; $n=3$ ). The result of a typical experiment is shown. $\boldsymbol{H}$, Shown is the percentage of NPY-mRFP-labeled SGs that were also decorated with a GFP-MyoVa tail, a construct comprising the globular tail of MyoVa and the exons $A, C, E$, and F. The targeting of the GFP-MyoVa tail to SGs was reduced by Myrip silencing and rescued by expressing a siRNA1-insensitive Myrip construct (rescue) ( $p<0.0001$, ANOVA). Asterisks refer to a Tukey's post-test; ${ }^{* * *} p<0.001 ; n=15$ cells from two experiments. Cells expressing the rescue construct were identified using an anti-c-Myc antibody and an Alexa-350-labeled secondary antibody. Noteworthy is that the expression of the GFP-MyoVa tail was not reduced by Myrip silencing. I, J, Moreover, in $\sim 60 \%$ of Myrip-siRNA1-treated cells, the GFP-MyoVa tail exhibited a marked soluble cytosolic pattern without any visible enrichment on intracellular structures. $K$, Coimmunoprecipitation of Myrip and myosin Va. Coimmunoprecipitation of the GFP-MyoVa tail with myc-tagged Myrip after expression in BON cells is shown. Immunoprecipitation was done with an anti-myc antibody and immunoblotting with anti-MyoVa antibodies. One-fifteenth of cell extracts and one-fourth of immunoprecipitates were analyzed. The positions of endogenous MyoVa and the MyoVa tail are shown by an arrowhead and an arrow, respectively. Scale bars: $5 \mu \mathrm{m}$.

\section{Myrip promotes the peripheral retention of SGs}

To determine whether the decreased SG density beneath the PM was due to impaired delivery or retention of SGs, we designed an assay to measure the time spent by SGs in the evanescent field. A $405 \mathrm{~nm}$ laser diode was coupled to our TIRF microscope and used to photoconvert SG-targeted NPY-Dendra2. A short UV illumination induced a green-to-red shift of the fluorescence emission of Dendra2 in the subset of SGs present in the evanescent field (Fig. $4 A, B$ ). In agreement with the effect of Myrip on SG density in the TIRF area (Fig. 2D), Myrip-FL, but not Myrip- $\Delta \mathrm{C}$, increased the integrated fluorescence of NPY-Dendra2 measured immediately after photoconversion (data not shown). In con- trast, Myrip knockdown reduced this value. Both the fluorescence intensity (Fig. 4C) and the number of visible SGs (Fig. 4D) decreased over time. The decay is likely to reflect SG motion toward the cell interior since under resting conditions secretion is extremely low and cannot contribute to the observed fluorescence decay. Myrip-FL slowed the decay of both the number of labeled SGs and fluorescence intensity, whereas Myrip siRNAs accelerated it, indicating that Myrip promotes the retention of SGs at the cell periphery. Interestingly, Myrip- $\Delta C$ slowed the decay of the number of SGs kept in the evanescent field but accelerated the fluorescence loss, suggesting that the overexpression of Myrip- $\Delta$ C increased the distance of the SGs to 
A
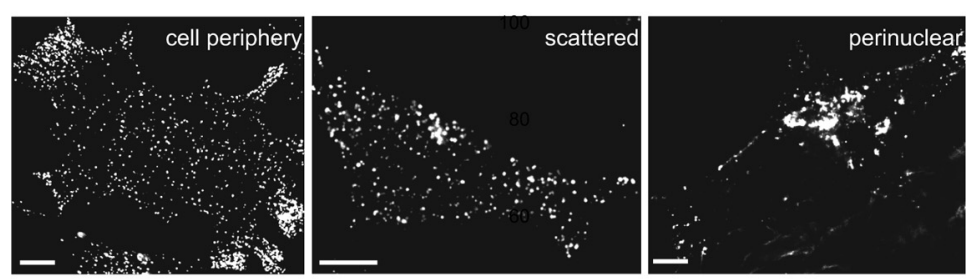

B

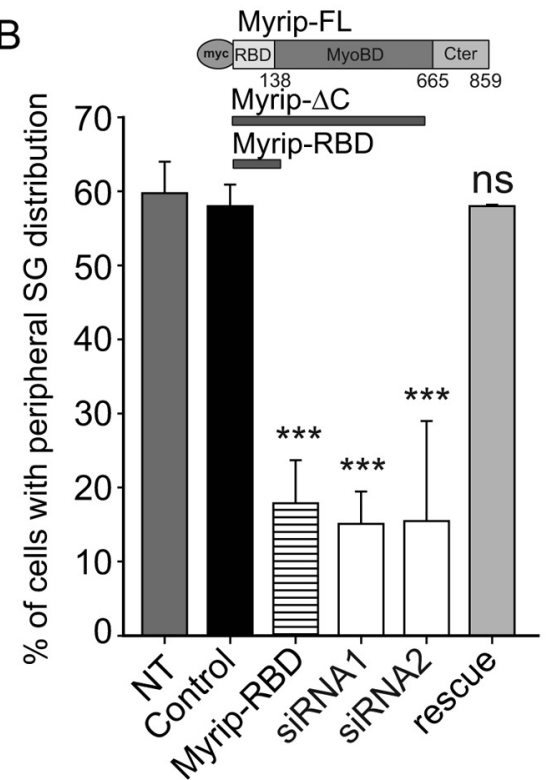

D

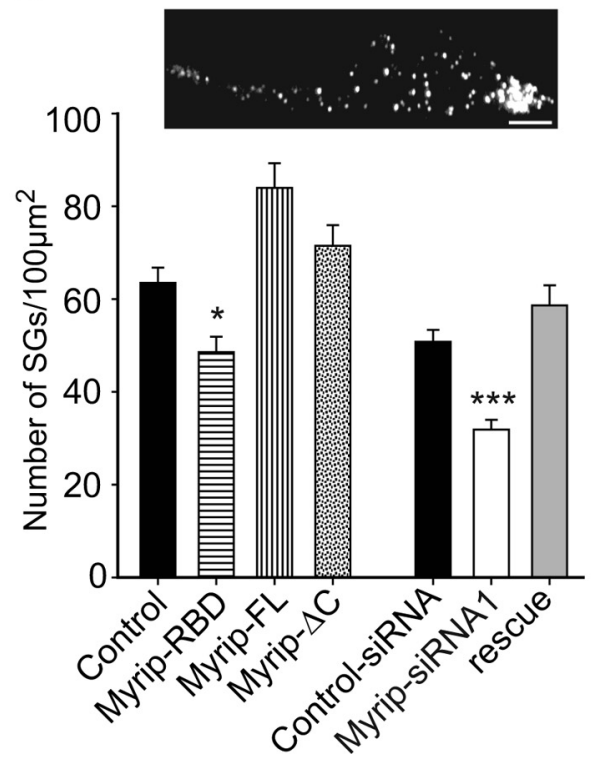

C

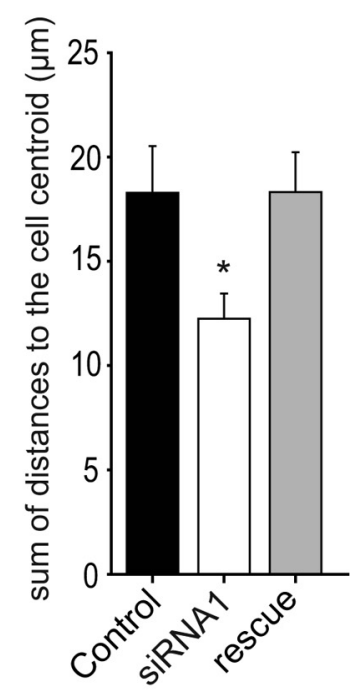

E

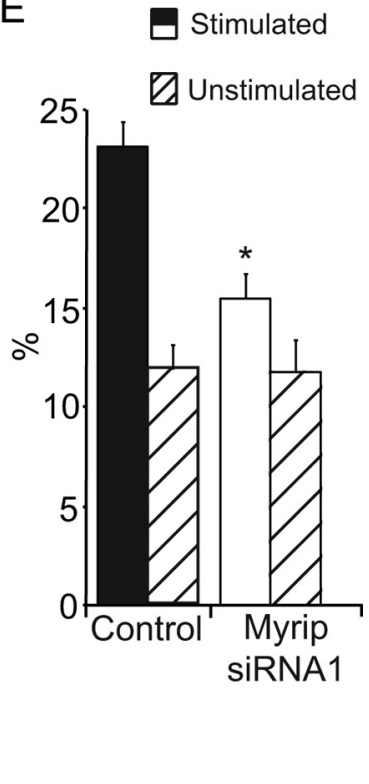

the PM while keeping them at the cell periphery. An increased photobleaching of SGs could also account for the higher fluorescence loss observed upon expression of Myrip- $\Delta$ C. SGs would bleach faster if they were closer to the PM and therefore exposed to a greater flux of photons. However, this possibility seems unlikely because the initial fluorescence intensity of individual SGs was not modified by Myrip- $\Delta \mathrm{C}$ expression, as would be the case if their distance to the PM was lower (data not shown).

Using TIRF imaging, we also counted the number of NPY-GFP-labeled SGs that entered or left the evanescent field during time series of TIRF images (Fig. 5A). We observed that knocking down Myrip (Fig. $5 B$ ) increased both the rates of SG appearance and disappearance. MyoVa silencing induced similar, but less pronounced, effects (Fig. 5C). The increased rate of SG appearance may result from the higher density of SGs in the cell center observed in Myrip knockdown cells. In contrast, the increased rate of $S G$ disappearance cannot be accounted for by changes in the number of SGs participating in the trafficking, but rather reflects an increase in the rate constant that controls the movement of SGs from the actin-rich layer to the cell center (see the Discussion section for a discussion of these data within the framework of the model depicted in Fig. 10; for the quantification of the different kinetic parameters, see Table 1).

\footnotetext{
$\leftarrow$

graph shows the mean ( \pm SE) of the values obtained in 10 cells for each condition. $p=0.0388$ (ANOVA). D, Myrip controls the juxtamembrane SG density. BON cells were transfected with an empty vector (Control, 39 cells from 2 experiments) or a control siRNA duplex (Control-siRNA, 107 cells from 7 experiments), with vectors encoding Myrip-RBD (42 cells from 2 experiments), Myrip-FL, (38 cells from 2 experiments), Myrip- $\Delta$ C (38 cells from 2 experiments), or with Myrip-siRNA1 ( 96 cells from 7 experiments) and a Myrip rescue construct ( 20 cells from 2 experiments); they were also cotransfected with pNPY-mRFP to label SGs. Cells were imaged by TIRFM. Inset, A representative TIRFM image of a BON cell expressing NPYmRFP. Data are expressed as the mean $\pm S E$ of values obtained in the different cells; $p<0.0001$ (Kruskal-Wallis). $E$, Effect of Myrip silencing on secretion. BON cells were treated with control siRNAs or with Myrip-targeting siRNAs. Three days later, they were loaded with $\left[{ }^{3} \mathrm{H}\right]$ serotonin, washed, and stimulated with Locke's solution supplemented with $3.7 \mathrm{~mm}$ $\mathrm{Ba}^{2+}$ (black and white bars) for 10 min or incubated in nonstimulating bathing medium for the same duration (dashed bars). Released serotonin was measured in the extracellular medium and expressed as a percentage of serotonin contained in the cells (mean \pm SE of $6-15$ wells). Scale bars: $5 \mu \mathrm{m}$. ${ }^{*} p<0.05 ;{ }^{* * *} p<0.001$
}

Figure 2. Myrip controls the distribution of SGs and their recruitment at the cell periphery. $A, B O N$ cells were transfected with S cellular extensions (left). However, upon Myrip silencing or Myrip-RBD expression, SGs are frequently scattered in the cell (middle) or concentrated in the perinuclear region (right). $\boldsymbol{B}$, Shown are the mean ( $\pm \mathrm{SE}$ ) percentages of cells with a marked enrichment of SGs at the cell periphery observed in control conditions (NT, nontransfected cells; $n=139$ cells from 4 independent experiments; control, $n=255$ cells from 6 experiments), upon expression of Myrip-RBD ( $n=109$ cells from 4 experiments), and upon transfection of Myrip-siRNA1 ( $n=190$ cells from 6 experiments), Myrip-siRNA2 ( $n=125$ cells from 3 experiments), and MyripsiRNA1 plus an myc-tagged siRNA1-insensitive Myrip construct (rescue; $n=81$ cells from 2 experiments). Inset, schematic representation of the Myrip constructs used in this study. $p<0.0001$ (ANOVA); ${ }^{* * *} p<0.001$ (Tukey's post-test); ns, nonsignificant. C, To quantify the cellular distribution of SGS, epifluorescence images of NPY-GFP-expressing cells were segmented using MIA software. For each cell, the mean distance of the thresholded pixels to the centroid of the cell footprint was computed. The 


\section{Myrip silencing interferes with SG motion along microtubules}

To determine how Myrip controls the retention of SGs at the cell periphery, we characterized SG mobility in the subplasmalemmal region by TIRF imaging and single-particle tracking. The mean lateral diffusion coefficient $\left(D_{x y}\right)$, an index of the mobility of SGs, on entire trajectories was significantly higher in Myrip-silenced cells $\left(D_{x y}=92.3 \pm 7.2 \times 10^{-4} \mu \mathrm{m}^{2} \cdot \mathrm{s}^{-1}\right.$; mean \pm SE; 653 SGs from 10 cells) than in control cells $\left(D_{x y}=24.3 \pm 5.2 \times 10^{-4}\right.$ $\mu \mathrm{m}^{2} \cdot \mathrm{s}^{-1}$; mean $\pm \mathrm{SE} ; 761$ SGs from 10 cells; $p=0.007)$. The distribution of mean $D_{x y}$ values computed on entire trajectories is shown in Figure $6 \mathrm{~A}$ and indicates two major effects of Myrip silencing: (1) an increased proportion of SGs with a very high $D_{x y}$ value $\left(>50 \times 10^{-4} \mu \mathrm{m}^{2} \cdot \mathrm{s}^{-1}\right)$ and (2) a $50 \%$ reduction in the percentage of almost-immobile SGs $\left(D_{x y}<5 \times 10^{-4}\right.$ $\left.\mu \mathrm{m}^{2} \cdot \mathrm{s}^{-1}\right)$.

To further characterize the effect of Myrip on fast-moving SGs, we manually selected subtrajectories characterized by fast $\left(D_{x y}>50 \times 10^{-4} \mu \mathrm{m}^{2} \cdot \mathrm{s}^{-1}\right)$ and directed motion. Such trajectories are severely reduced upon nocodazole treatment, indicating that they correspond to SGs moving along microtubules (Desnos et al., 2007b). The proportion of time spent in a directed motion was increased from $3.8 \pm 0.4 \%$ in control cells to $11.5 \pm$ $1.5 \%$ in Myrip knockdown cells (mean \pm SE; $p=0.002$ ). During these periods, SG velocity was $0.39 \pm 0.02 \mu \mathrm{m} \cdot \mathrm{s}^{-1}$ in control cells and $0.63 \pm 0.03 \mu \mathrm{m} \cdot \mathrm{s}^{-1}$ in Myrip-silenced cells (mean $\pm \mathrm{SE} ; p=$ 0.0002 ), and the distribution of velocity values was shifted to higher values by Myrip silencing (Fig. 6B). In addition, the length of microtubule-based runs was increased in Myrip knockdown cells $(1.49 \pm 0.11 \mu \mathrm{m})$ compared to control ones $(1.15 \pm 0.06$ $\mu \mathrm{m} ; p=0.04)$.

\section{Myrip promotes SG attachment to the plasma membrane}

Next, we focused on stalled SGs. While diffusing with an average $D_{x y}$ equal to $25 \times 10^{-4} \mu \mathrm{m}^{2} \cdot \mathrm{s}^{-1}$ in control conditions, SGs frequently display periods of highly restricted mobility with $D_{x y}$ values as low as $10^{-4} \mu \mathrm{m}^{2} \cdot \mathrm{s}^{-1}$. Several authors have suggested that these stalling periods correspond to the attachment of SGs to the PM (Huet et al., 2006; Desnos et al., 2007b; Nofal et al., 2007; Karatekin et al., 2008; Yizhar and Ashery, 2008; Ostrowski et al., 2010). Before analyzing the consequences of impairing Myrip on this class of SG motion, we first investigated further whether transient stalling could be used as a criterion of attachment to the PM. The fluctuations in SG mobility were monitored by measuring the variations of $D_{x y}$ along the SG trajectories, using a rolling analysis window, and immobilization periods were defined as portions of trajectories during which the $D_{x y}$ value droped below a threshold of $D_{\text {min }}=5 \times 10^{-4} \mu \mathrm{m}^{2} \cdot \mathrm{s}^{-1}$ (Fig. $7 A, B$ ). The choice of this threshold was driven by the observation that, within
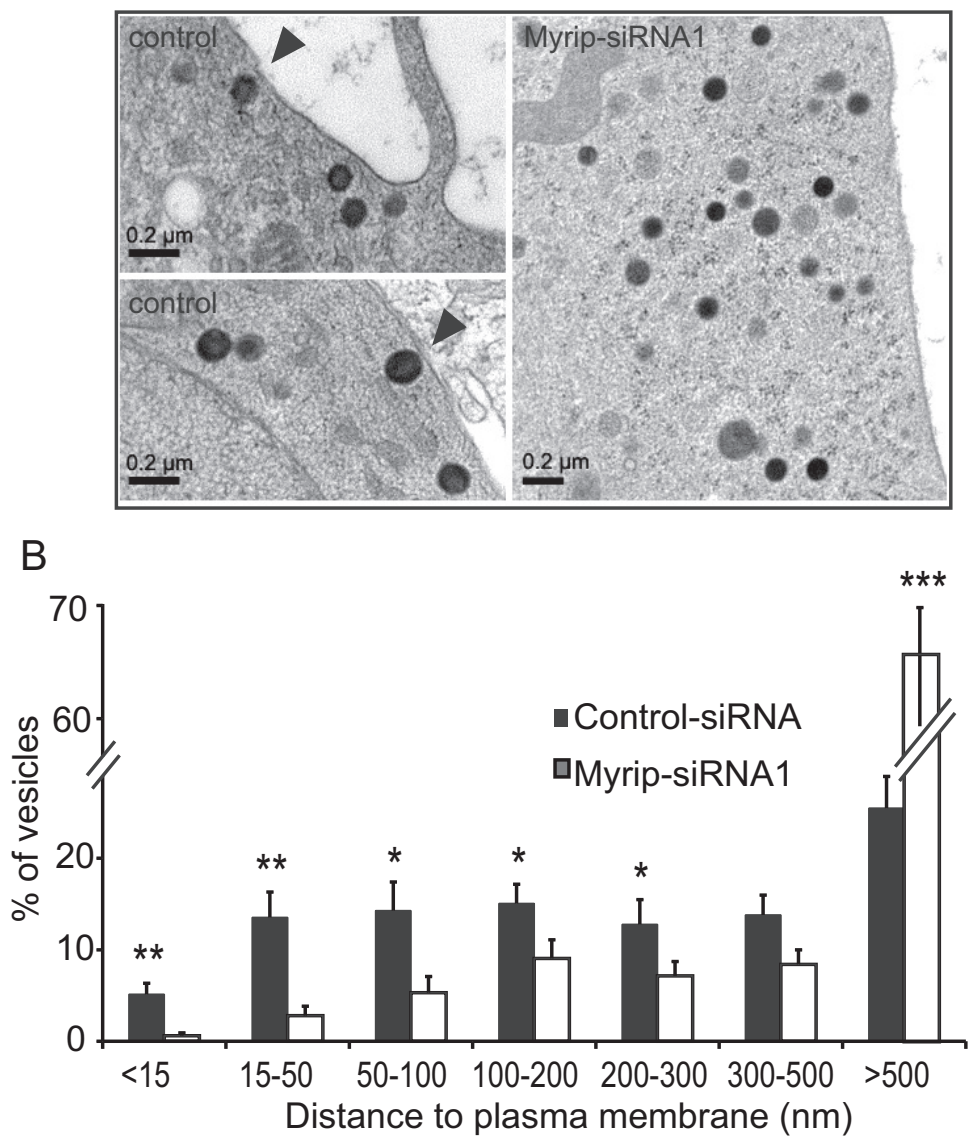

Distance to plasma membrane $(\mathrm{nm})$

Figure 3. Ultrastructural localization of SGs in BON cells. A, Ultrathin cryosections of BON cells. BON cells were treated with control siRNAs or with Myrip siRNAs as indicated, frozen under high pressure to protect the cytoarchitecture, and processed for Distros plasma membrane. B, Myrip silencing changed the localization of SGS. Dribution of the values of the distance between SGs and the PM expressed as percentage of the total number of SGs detected in each picture ( $n=30$ images). The data show that Myrip silencing reduced the number of SGs close to the PM $(<15 \mathrm{~nm}$ or located between 15 and $50 \mathrm{~nm}$ ) and increased the number of remote SGs $(>500 \mathrm{~nm}) .{ }^{*} p<0.05$; ${ }^{* *} p<0.01$; ${ }^{* * *} p<0.001$.

the $2 \mathrm{~s}$ preceding exocytosis, most of the SGs, which are probably attached to the PM, display a $D_{x y}$ value of $<5 \times 10^{-4} \mu \mathrm{m}^{2} \cdot \mathrm{s}^{-1}$ (Desnos et al., 2007b). However, due to the stochastic nature of Brownian motion, even purely diffusive trajectories characterized by an average diffusion coefficient of $10^{-3} \mu \mathrm{m}^{2} \cdot \mathrm{s}^{-1}$ may exhibit short periods during which $D_{x y}$ is lower than $D_{\text {min }}$. To distinguish such false positive stalling events from real periods of SG attachment, it was thus necessary to set a minimum crossing time, $t_{\text {min }}$. Therefore, we compared the distribution of immobilization times between SG tracks and simulated Brownian trajectories. The survival curves displayed on Figure $7 C$ show that with $t_{\min }$ set to $5 \mathrm{~s}$, false positive stalling events were contributing by $<1 \%$ to the events detected for SGs. Stalling periods were also detected on trajectories of latex beads diffusing in a glycerol/ water mix, and we confirmed that, with this minimum crossing time, the probability of detecting stalling events was at least 100 times higher for SGs than for beads (Fig. 7C). To further substantiate the fact that stalling periods reflect a constrained behavior, we compared the anomaly of the diffusion between stalling periods longer than $5 \mathrm{~s}$ and the rest of SG trajectories. The anomaly parameter $\alpha$ quantifies the restriction of the diffusion: the lower the value of $\alpha$, the more restricted the diffusion, with $\alpha$ ranging between 0 and 1 . The stalling periods were characterized by a strong restriction of SG motion, with $\alpha \approx 0.4$, in agreement with 
A
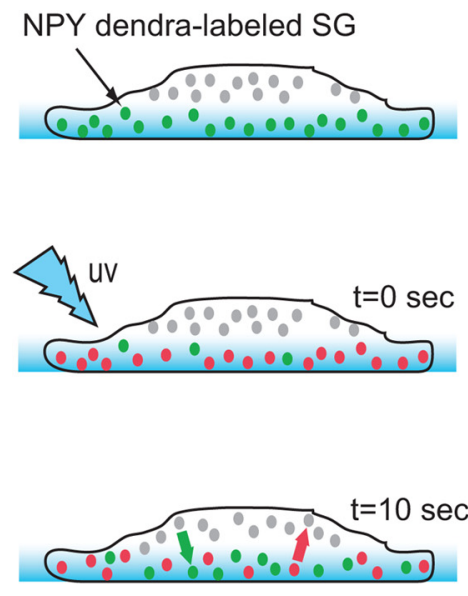

B
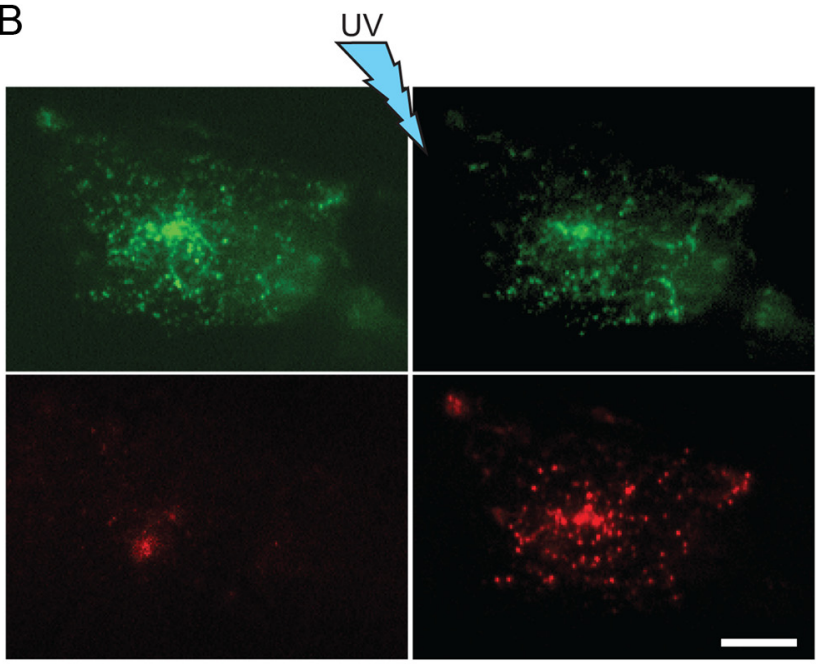

C

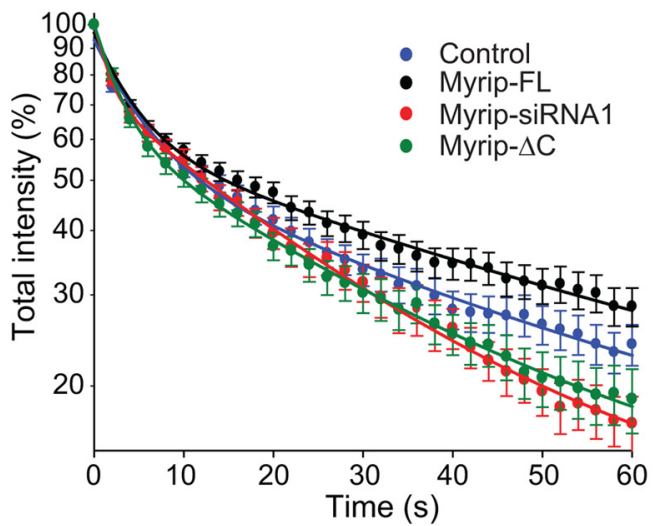

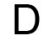

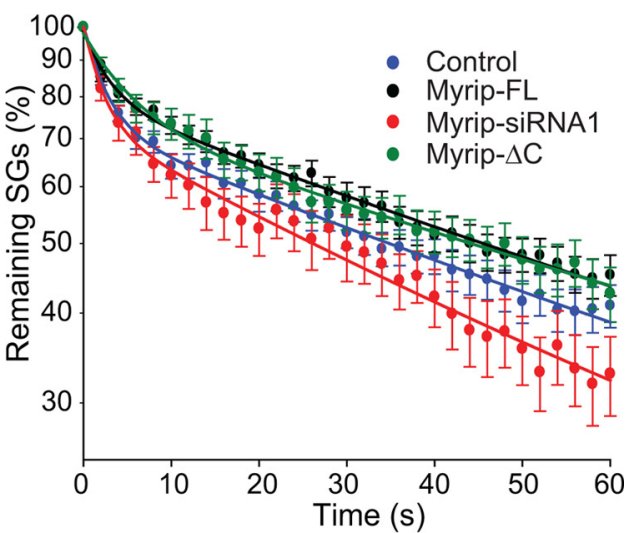

Figure 4. Myrip promotes the retention of SGs at the cell periphery. $A$, Principle of the experiment. Photoconversion of NPY-Dendra2 was used to label the subset of SGs that were close to the PM at time 0 (when a 2 s pulse of $405 \mathrm{~nm}$ light was delivered via the evanescent field) and to follow their dynamics over time. The signal is expected to decrease as photoconverted SGs leave the evanescent field area and are replaced by nonconverted ones. $\boldsymbol{B}$, Photoconversion of NPY-Dendra2-labeled SGs. TIRF images of a cell in the green and red channels before (left) and after (right) a pulse of $405 \mathrm{~nm}$ light are shown. Scale bar, $5 \mu \mathrm{m}$. C, Fluorescence decay depends on Myrip. Plotted is the mean ( $\pm \mathrm{SE}$ ) integrated intensity per $100 \mu \mathrm{m}^{2}$ of the red fluorescence over time expressed as a percentage of the value measured just after photoconversion (time $0 ; n=47$ control cells, 16 Myrip knockdown cells, 57 cells overexpressing Myrip-FL, and 31 cells overexpressing Myrip- $\Delta C$ ). $D$, The graph shows the number of red SGs counted on each frame and expressed as percentage of the initial value. The curves were fitted with the sum of two exponentials. The fit obtained for controls was significantly different from that obtained for Myrip-FL ( $p=0.0039$; extra sum of squares F test), Myrip knockdown $(p<0.0001)$, and Myrip- $\Delta C(p<0.0001)$.

an attachment to the PM. In contrast, outside these stalling periods, SG diffusion showed only moderate restriction with $\alpha \approx 0.8$ (Fig. 7D). Finally, using the fact that, by TIRF microscopy, changes in SG fluorescence intensity report changes in the position along the vertical axis, we measured the difference in the altitude of SGs between two successive periods of immobilization. We found that, in most of the cases, the difference was $<20$ $\mathrm{nm}$ (i.e., the resolution of our microscope in the $z$-axis) (Fig. 7E). The data thus suggest that immobile SGs are at the same altitude, i.e., at the PM. Survival curves for SG immobilization times were best fitted with two exponentials (Fig. 7C). It is noteworthy that both the short-lasting (characteristic time, $\sim 4 \mathrm{~s}$ ) and the longlasting (characteristic time, $\sim 15 \mathrm{~s}$ ) components were observed in single cells and thus cannot be attributed to the heterogeneity of the population of cells (Fig. 7C). If slow diffusion can contribute a significant fraction of the fast component of the survival curves, it is not the case of the slow component, which we therefore used as a refined criterion of SG attachment to the PM.

We reported previously that MyoVa promotes SG attachment to the PM (Desnos et al., 2007b), an effect that could be due to an increase in either the occurrence or the duration of attachment events. To resolve this issue, we analyzed the effect of MyoVa silencing on the distribution of immobilization times. MyoVa depletion reduced the occurrence of the long-lasting component but not its characteristic time $\tau_{2}$ (Fig. $7 G, I, J$ ). This reduction in the occurrence of SG immobilization was observed with three different MyoVa siRNAs, indicating that it was not due to off-target effects. We conclude that MyoVa promotes the recruitment of SGs to the PM rather than the stability of the immobilized state.

Similarly, Myrip silencing reduced the occurrence of the longlasting component (Fig. $7 \mathrm{H}, \mathrm{K}$ ). In addition, it reduced the characteristic time, $\tau_{2}$, of this immobilization process (Fig. $7 H, L$ ). It is noteworthy that survival curves were rescued by expressing a siRNA-insensitive Myrip construct arguing against an off-target effect of the siRNAs. Since Myrip silencing also reduced the number of SGs present in the TIRF area (Fig. 2D), its overall effect was to diminish the number of attached SGs per surface area by $\sim 65 \%$. This conclusion is consistent with the data obtained by EM showing a severe reduction in the number of SGs morphologically attached to the PM in Myrip knockdown cells and accounts for the secretory defects observed in these cells. 
A

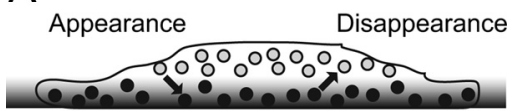

$\mathrm{B}$
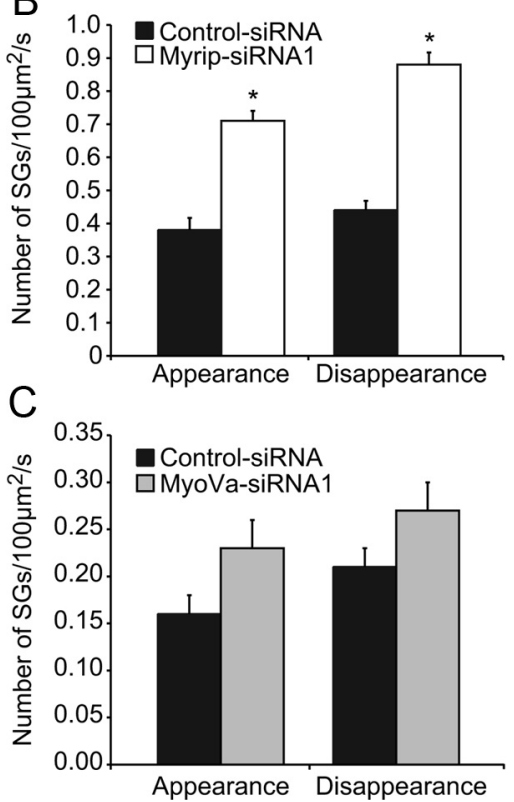

Figure 5. Effect of Myrip and MyoVa on the delivery of SGs at the cell periphery. $A$, Principle of the experiment. NPY-labeled SGs were imaged by TIRFM. Their appearance in the TIRF area and their disappearance were manually monitored along time-series images. B, Quantification of the Myrip data. Myrip silencing increased the occurrence of $S G$ arrival at the cell periphery and also the disappearance of SGs. The increase in SG appearance does not imply that Myrip silencing promotes $S G$ delivery at the cell periphery (i.e., an increase in $k_{1}$; see model in Fig. 10), but rather reflects the increase in the density of SGs in the cell center (we actually see an increase in $k_{1}$ C). ${ }^{*} p<0.05$; Mann-Whitney test; $n=10$ cells from 2 independent experiments. C, Quantification of the MyoVa data (same as in $\boldsymbol{B}$, but for MyoVa silencing; $n=20$ cells from two independent experiments). The effect of MyoVa knockdown is not as strong as that of Myrip $\operatorname{knockdown}(p=0.07)$

\section{Myrip restricts the diffusion of SGs within the} actin-rich cortex

Next, we characterized the effect of Myrip on the mobility of SGs when they were neither stalled nor moving along microtubules (see Materials and Methods). During such periods, SGs display Brownian motion and are therefore diffusing with or within the actin-rich cortex (Fig. 7D) (Huet et al., 2006). Both Myrip and MyoVa silencing increased the proportion of time spent by SGs in the diffusive state (Fig. 8A) and the mobility of SGs in the diffusive state (Myrip knockdown cells, $D_{x y}=22.6 \pm 1.3 \times 10^{-4}$ $\mu \mathrm{m}^{2} \cdot \mathrm{s}^{-1}$ vs control, $14.9 \pm 1.9 \times 10^{-4} \mu \mathrm{m}^{2} \cdot \mathrm{s}^{-1} ; 10$ cells, $p=$ 0.01; MyoVa-silenced cells, $D_{x y}=24.5 \pm 2.9 \times 10^{-4} \mu \mathrm{m}^{2} \cdot \mathrm{s}^{-1}$ vs control, $14.0 \pm 1.5 \times 10^{-4} \mu \mathrm{m}^{2} \cdot \mathrm{s}^{-1} ; 16$ cells, $\left.p=0.003\right)$. These results suggest that the Rab27/Myrip/myosin Va complex restricts the mobility of SGs within the actin-rich cell cortex, most likely by tethering SGs to actin filaments, to maintain SGs in the vicinity of release sites.

\section{The C-terminal domain of Myrip participates in SG} attachment to the plasma membrane

The above-mentioned difference between the effects of Myrip and MyoVa silencing on the duration of long-lasting SG immobilization suggested that Myrip may exert MyoVa-independent actions. To investigate this possibility, we overexpressed Myrip, Myrip- $\Delta \mathrm{C}$ (which can interact with MyoVa), or Myrip-RBD
A

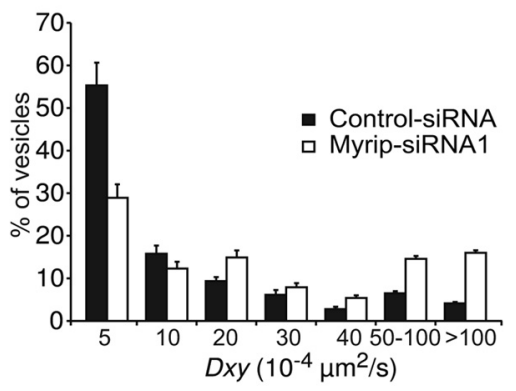

$\mathrm{B}$

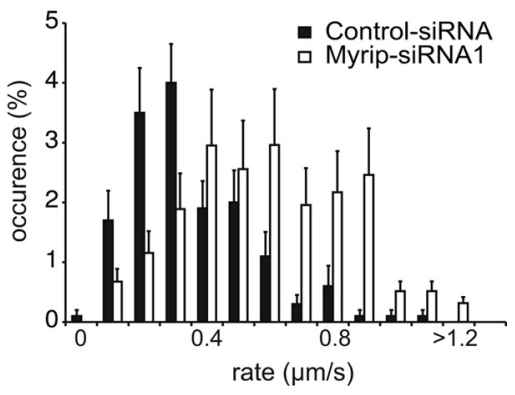

Figure 6. Myrip silencing changes the mobility of SGs. Single SGs were imaged by TIRFM under resting conditions and tracked in BON cells transfected with siRNA duplexes (control, black bars; Myrip siRNA, white bars) and pNPY-GFP. $A$, Shown is the distribution of $D_{x y}$ values computed on entire trajectories ( $n=10$ cells from 2 independent experiments; control, 761 SGs; Myrip-siRNA1, 653 SGs). Myrip silencing significantly reduced the percentage of SGs with $D_{x y}<5 \times 10^{-4} \mu \mathrm{m}^{2} \cdot \mathrm{s}^{-1}$ that are likely attached to the PM and increased the proportion of SGs with $D_{x y}>50 \times 10^{-4} \mu \mathrm{m}^{2} \cdot \mathrm{s}^{-1}$ that are likely moving along microtubules. $\boldsymbol{B}$, Myrip silencing increases $S G$ velocity on microtubules. Subtrajectories corresponding to directed motion (i.e., along microtubules) were selected. Shown is the distribution of SG velocities in control cells (filled bars; 10 cells, 155 periods of directed motion) or in Myrip knockdown cells (open bars; 10 cells, 250 periods of directed motion). The distribution is shifted to the right upon Myrip silencing.

(which does not bind to MyoVa) into BON cells and measured its effect on SG mobility. Overexpression of Myrip-RBD or Myrip- $\Delta \mathrm{C}$ increased the mean diffusion coefficient, $D_{x y}$, computed on entire trajectories (Fig. $9 A$ ) and reduced the percentage of SGs with very low $D_{x y}$ values $\left(<5 \times 10^{-4} \mu \mathrm{m}^{2} \cdot \mathrm{s}^{-1}\right)$ by $\sim 35 \%$ compared to control cells or to cells expressing Myrip-FL $(p<$ 0.0001 , ANOVA). We then measured the duration of immobilization events along trajectories and found that Myrip-RBD and Myrip- $\Delta \mathrm{C}$ reduced the occurrence of long-lasting immobilization periods by 53 and $27 \%$, respectively. Myrip- $\Delta C$ also decreased the characteristic time of long-lasting immobilization compared to Myrip-FL (Fig. 9B) $(p=0.0004)$, suggesting that the C-terminal region of Myrip contributes to the stability of SG attachment at the plasma membrane.

\section{Discussion}

In this study, we identified Myrip as a factor that couples the capture of SGs in the actin-rich cell cortex and their attachment to the PM, a process mandatory for exocytosis. Myrip thus plays a major role in the accumulation of SGs at the cell periphery and in the replenishment of the releasable pool of SGs.

\section{Inferring the functional state of SGs from their mobility}

To understand SG recruitment at release sites, our approach was to track single SGs, to analyze their motion along trajectories and to infer their functional state from their behavior. Previous studies have shown the existence of three classes of SG motion and 
A
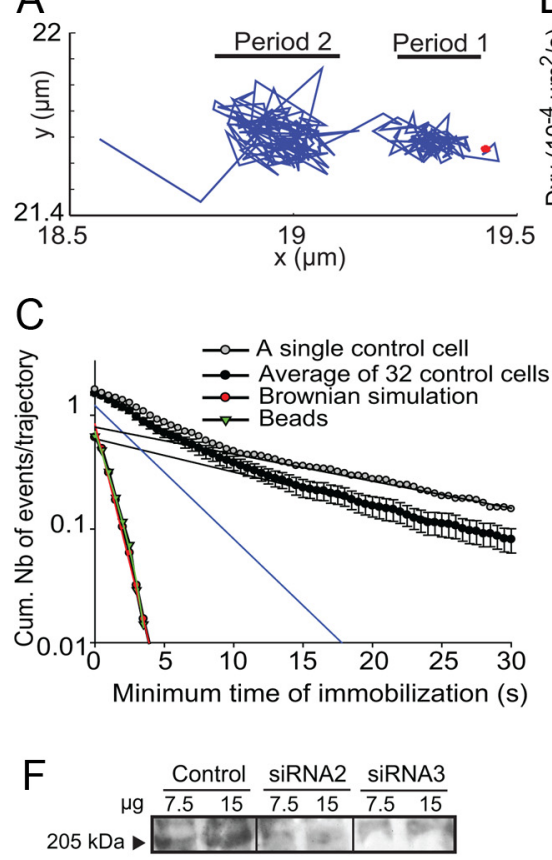

G

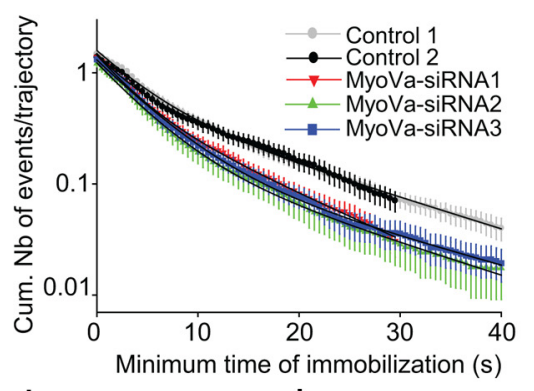

I

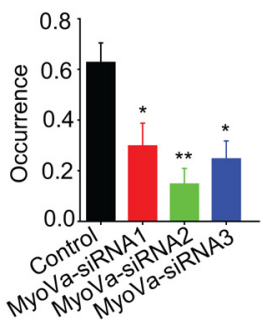

B

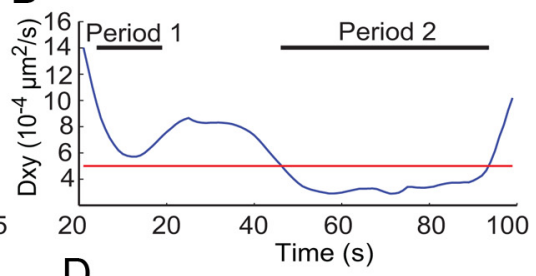

D

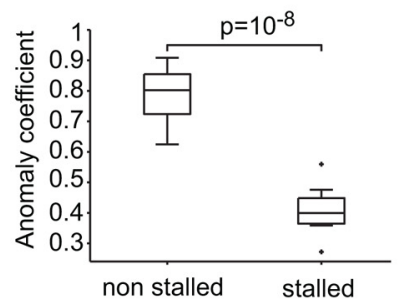

E

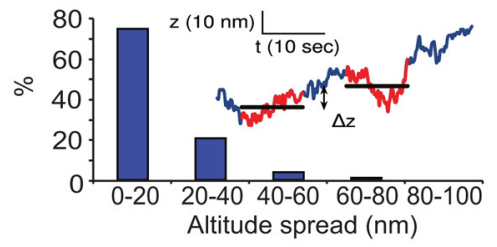

$\mathrm{H}$

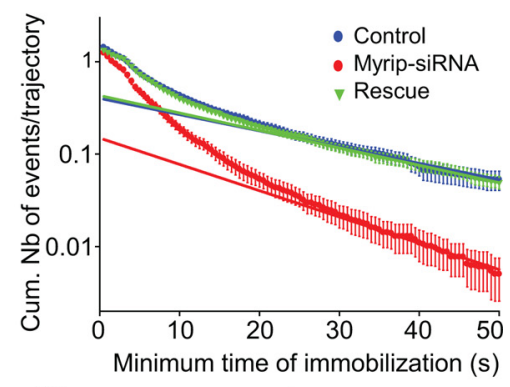

$\mathrm{K}$
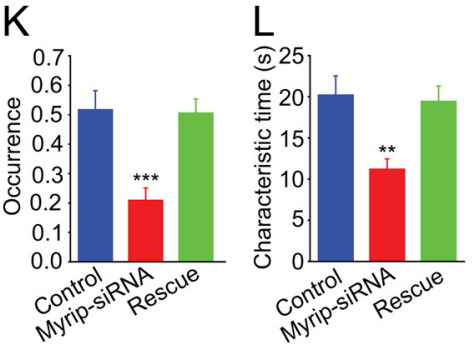

Figure 7. Myrip promotes $S G$ attachment to the plasma membrane. $A, B$, Example of a single $S G$ trajectory and detection of immobilization periods. The $x-y$ displacement of a single $S G$ is shown in $A$. The initial point of the trajectory is depicted by a red dot. The $D_{x y}$ values were computed along the trajectory using a rolling analysis window and plotted against time in $\boldsymbol{B}$. Below a threshold value $D_{\min }=5 \times 10^{-4} \mu \mathrm{m}^{2} \cdot \mathrm{s}^{-1}$ (red line), SGs were considered immobile. In this example, the SG displayed a constrained behavior during two subtrajectories. Period 2 was categorized as an immobilization period. $C$, The cumulative distribution of the number of immobilization events identified in control trajectories (filled circles, 32 cells) is plotted as a function of the minimum duration of immobilization. The best fit was obtained with the sum of two exponentials, indicating the existence of two different processes (black line, slow component; blue line, fast component). A similar distribution is found in single cells; an example is shown in gray. Ninety-nine percent of the immobilization periods detected in simulated Brownian trajectories (red) and fluorescent bead trajectories (green) were shorter than $5 \mathrm{~s}$, and their distribution can be fitted by a single exponential. $D$, Box plot of the anomaly coefficient, which reports the restriction of $\mathrm{SG}$ diffusion. The coefficient has a value of 1 in purely diffusive trajectories. Five hundred and eighteen stalled periods (longer than $5 \mathrm{~s}$ ) and 396 nonstalled periods from 10 cells were analyzed. $\boldsymbol{E}$, The trace shows the motion of a single $S G$ along the $z$-axis. Sections of the trajectory depicted in red correspond to periods of immobilization in the $x-y$ plane. Horizontal bars report the mean altitude of the SG during these two periods. The histogram shows the distribution of the $\Delta z$ values between successive immobilization periods, as measured in 114 trajectories from seven cells. $\boldsymbol{F}$, Knockdown of MyoVa expression. Three days after transfection of siRNAs, MyoVa levels were analyzed by immunoblotting in cells treated with a contro siRNA, MyoVa-siRNA2, or MyoVa-siRNA3. The result of a typical experiment is shown. For MyoVa-siRNA1 see the study by Desnos et al. (2007b). G, MyoVa silencing decreases the occurrence but not the duration of docking periods. BON cells were transfected with NPY-GFP and siRNAs targeting MyoVa and imaged by TIRFM ( $n=32$ control cells, 19 MyoVa-siRNA1 cells, 11 MyoVa-siRNA2

suggested that (1) rapid and asymmetric trajectories correspond to SGs moving along microtubules, (2) stalled SGs are attached to the PM, and (3) the remaining trajectories correspond to random walks within the actin-rich cell cortex (Huet et al., 2006; Desnos et al., 2007b).

One difficulty is to distinguish random walks from stalling periods. Here, we used a method that combined a selection of immobilization periods characterized by a low $D_{x y}$ value with the analysis of their durations to provide an index of SG confinement. Analyzing the distribution of immobilization times helped to distinguish attachment (the long-lasting component of the survival curves) and slow random walk. Subtrajectories selected by $D_{x y}$ thresholding and longer than 5 s were characterized by a nonlinear relationship between MSD and time, indicating a subdiffusive behavior. This is in agreement with our previous finding that these periods feature a negative autocorrelation of the motion along the $z$-axis, suggesting that during these periods, SGs do not move freely but are attached (Desnos et al., 2007b). SG immobilization could result from binding to other structures than the PM, especially to actin filaments. However, actin structures are not immobile (Desnos et al., 2007b) (our unpublished observations), and thus cannot support stable SG immobilization. Furthermore, we found that SGs that un-

\section{$\leftarrow$}

cells, and 12 MyoVa-siRNA3 cells from 3 independent experiments with 1500 - 4000 trajectories per condition). Immobilization periods were detected as described in $\boldsymbol{B}$. Shown is the logarithmic representation of the cumulative distribution of the number of immobilization events plotted as a function of their duration. The mean duration of trajectories was $\sim 16 \mathrm{~s}$ in control cells and $\sim 13$ s in MyoVa knockdown cells. $\boldsymbol{H}$, Myrip silencing decreases both the occurrence and the duration of docking periods (same as in $\mathbf{G}$ but for Myrip knockdown cells; $n=32$ control cells, 30 Myrip-siRNA1 cells, and 20 rescue cells from 2-4 independent experiments, with 2500 - 3000 trajectories per condition). Note that both the intercept and the slope of the slow component of the distribution are affected by Myrip silencing. The mean duration of trajectories was $\sim 18 \mathrm{~s}$ in control cells, $\sim 12$ s in Myrip knockdown cells, and $\sim 16$ s in rescue cells. $I-L$, The histograms show the values (mean $\pm S E$, calculated in each cell and averaged over the different cells) of the occurrence (i.e., the intercept of the fit curve with the $y$-axis) and the characteristic time of the slow component of the distribution of immobilization times; $p=0.0006$ (MyoVa, occurrence), $p=0.98$ (MyoVa, characteristic time), $p<$ 0.0001 (Myrip, occurrence), and $p=0.0017$ (Myrip, characteristic time; Kruskal-Wallis test). Asterisks indicate the results of a Dunn's multiple comparison test. Similar values were obtained by fitting the averaged distribution of immobilization times shown in $\mathbf{G}$ and $\boldsymbol{H} .{ }^{*} p<0.05 ;{ }^{* *} p<0.01 ;{ }^{* * *} p<$ 0.001 . 
A
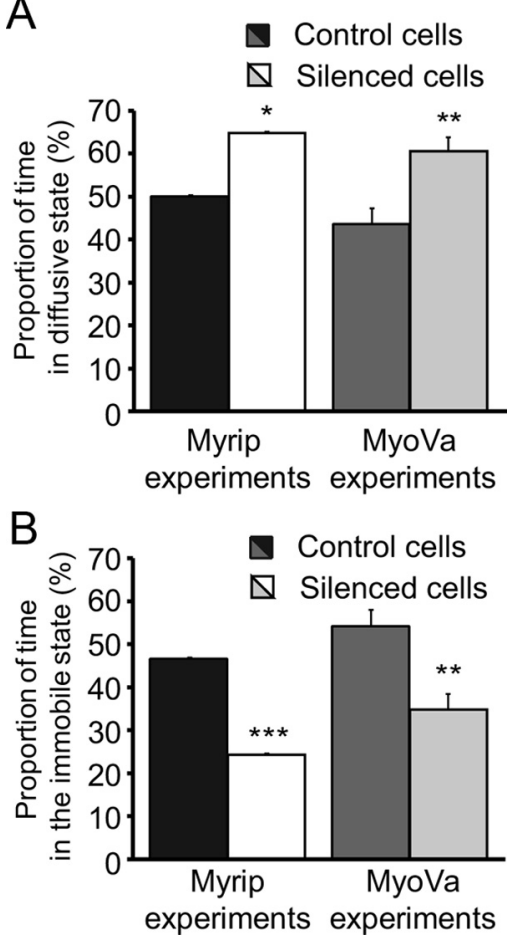

Figure 8. Effect of Myrip and MyoVa on the time spent in diffusive or immobile states. BON cells were transfected with siRNA duplexes (control, black; Myrip siRNA, white; MyoVa siRNA, gray) and pNPY-GFP and imaged by TIRFM. $D_{x y}$ values were computed along single trajectories. Subtrajectories corresponding to directed movements (manually selected) or to stalled periods $\left(D_{x y}<5 \times 10^{-4} \mu \mathrm{m}^{2} \cdot \mathrm{s}^{-1}\right.$ for $>6-8.5 \mathrm{~s}$ according to the conditions; see Materials and Methods) were identified. The remaining parts of the trajectories were considered diffusive. $A$, $\boldsymbol{B}$, Proportion of time spent in the diffusive $(\boldsymbol{A})$ or immobile $(\boldsymbol{B})$ state for SGs from control, Myrip knockdown cells, and MyoVa knockdown cells (10-20 cells per condition). The mean length of trajectories was $\approx 17 \mathrm{~s}$ in control cells, $14 \mathrm{~s}$ in MyoVa knockdown cells, and $10 \mathrm{~s}$ in Myrip knockdown cells. ${ }^{*} p<0.05 ;{ }^{* *} p<0.01 ;{ }^{* * *} p<0.001$.

dergo multiple immobilization periods during their trajectory generally immobilize at the same altitude. As actin filaments are present at different altitudes, this observation also suggests that SGs immobilize at the PM. We think that this approach provides a robust criterion of $S G$ attachment to the PM.

SG mobility is high in BON cells compared to primary neuroendocrine cells such as adrenal chromaffin cells and pancreatic $\beta$ cells (Degtyar et al., 2007; Michael et al., 2007). As we can collect many examples of the different possible behaviors and transitions between different classes of motion, this particular feature of BON cells facilitates the analysis of SG recruitment at exocytotic sites.

\section{A compartment model to analyze Myrip-dependent SG trafficking}

Our data indicate that Myrip has profound effects on SG distribution, mobility, and membrane attachment. To evaluate the different parameters controlling SG dynamics at the cell cortex, we designed a simple compartment model to describe SG delivery to the PM (Fig. 10A). The model posits that SGs are transported from the cell center (C) to the cell periphery (most likely along microtubules) and then enter a thin ( $\sim$ S S diameter) actin-rich layer (A) into which they diffuse until they find an attachment site at the PM (D). As suggested by Huet et al. (2006), we considered that the transition $\mathrm{C} \rightarrow \mathrm{D}$ is not allowed, i.e., that SGs coming from the cell center cannot reach the PM directly. The values of A $\left(0.22 \pm 0.025 \mathrm{SGs} / \mu^{2}{ }^{2}\right)($ Table 1$)$ and $\mathrm{D}(0.22 \pm 0.025 \mathrm{SGs} /$
A
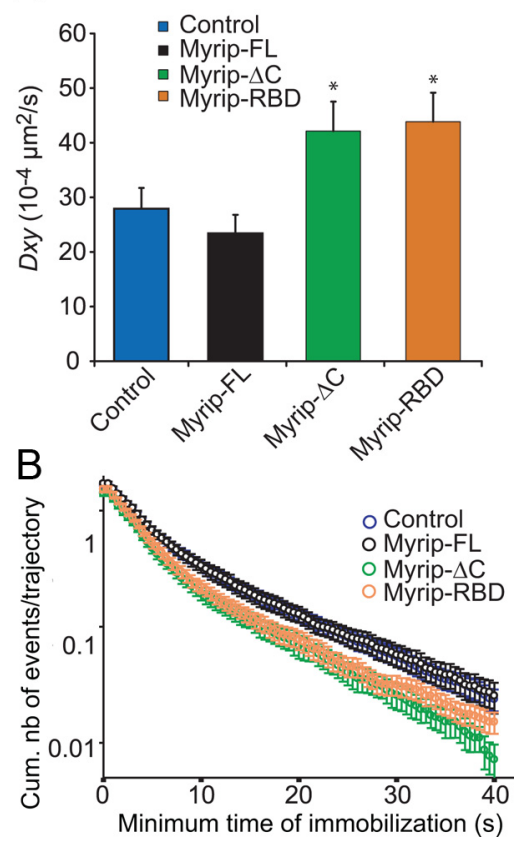

Figure 9. The C-terminal domain of Myrip plays a role in SG immobilization. $A$, Effect of Myrip constructs on overall SG mobility. BON cells were transfected with NPY-GFP and MyripRBD, Myrip-FL or Myrip- $\Delta$ C and imaged by TIRFM. SG trajectories were tracked, and $D_{x y}$ values were computed on entire trajectories. Shown are the means ( \pm SE) of the values obtained for 34 control cells (from 4 experiments) or 30 cells overexpressing Myrip constructs ( $n=3$ experiments). $p=0.0011$ (Kruskal-Wallis test). $\boldsymbol{B}$, Truncated Myrip constructs reduced long-lasting $S G$ immobilization. A rolling window was used to calculate $D_{x y}$ values along $S G$ trajectories and to detect immobilization portions of trajectories with a $D_{x y}$ value inferior to $5 \times 10^{-4}$ $\mu \mathrm{m}^{2} \cdot \mathrm{s}^{-1}$. Shown is the logarithmic representation of the cumulative distribution of the number of immobilization events plotted as a function of their duration. The occurrence of the long-lasting component was lower in Myrip-RBD- and Myrip- $\Delta$ C-expressing cells than in control cells or Myrip-FL-expressing cells ( $p<0.0002$, extra sum of squares $F$ test). The slope of this component was also higher in Myrip- $\Delta C$ - than in Myrip-FL-expressing cells $(p=0.0004)$. ${ }^{*} p<0.05$.

$\mu \mathrm{m}^{2}$ ) were derived from the density of SGs observed by TIRFM (density $=\mathrm{A}+\mathrm{D})$ and from the proportion of the time spent in the stalled or diffusive states, as deduced from the analysis of $D_{x y}$ along trajectories (Figs. 7, 8). At steady state, the number of SGs arriving in the TIRF area should be similar to the number of SGs exiting the TIRF field; hence, $k_{-1} \mathrm{~A}=k_{1} \mathrm{C}$. Consistently, SG appearance and disappearance rates were found to be similar (Fig. $5)$. From the rate of SG disappearance $\left(k_{-1} \mathrm{~A}\right)$ and the measured value of A, we obtained $k_{-1}=0.016 \pm 0.002 \mathrm{~s}^{-1}$. Similarly, at steady state, the number of SGs diffusing in the cell cortex that immobilize at the PM is compensated by a similar number of SGs that come apart. Thus, $k_{2} \mathrm{~A}=k_{-2} \mathrm{D}$. The detachment rate constant $k_{-2}$ determines the characteristic lifetime, $\tau$, of the immobilized state $\left(\tau=1 / k_{-2}\right)$, which we inferred from the survival curves shown in Figure 7 (number of stalled SGs still immobilized after a time $t$ ). The exponential fit to the slow component of these curves gave a value for $k_{-2}\left(k_{-2}=0.056 \pm 0.007 \mathrm{~s}^{-1}\right)$, and hence for $k_{2}$, the rate constant of SG attachment to the PM $\left[k_{2}=k_{-2} \mathrm{D} /\right.$ $\left.\mathrm{A}=0.082 \pm 0.012 \mathrm{~s}^{-1}\right]$.

Using this compartment model, we simulated the photoconversion experiment shown in Figure 4 (Fig. 10 B). Both the experimental and simulated curves display a biphasic behavior which can be well fitted with the sum of two exponential components. The fast one (characteristic time, $6.5 \mathrm{~s}$, compared with $5.9 \mathrm{~s}$ for the experimental curve) represents the equilibration of $A$ and $D$ 
A

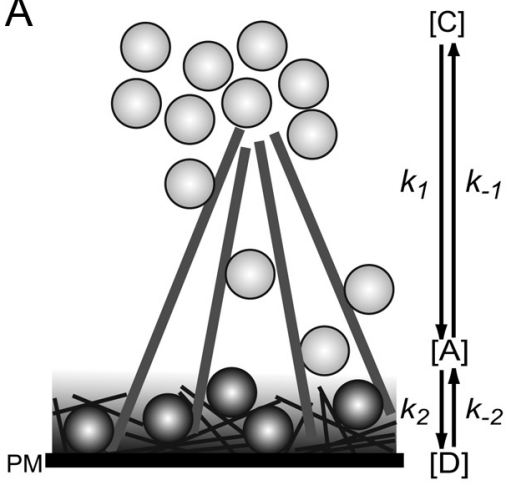

B

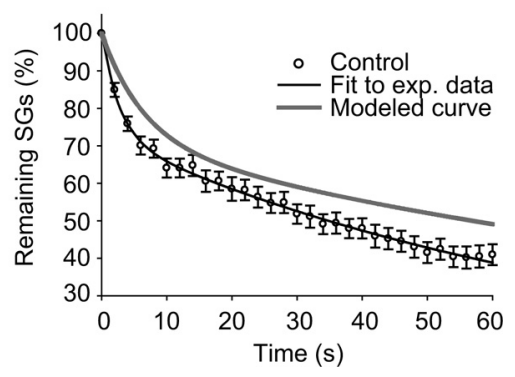

Figure 10. Model of SG delivery at the cell periphery. $A$, SGs may be attached to the PM (D) or located in the actin-rich juxtamembrane region (A) or in the cell center (C). Straight yellow lines represent microtubules along which $\mathrm{SG}$ s travel to reach or to leave the cell periphery. Thin lines represent the actin filament network. Transitions between the three states $C, A$, and $D$ are governed by the constants $k_{1}, k_{-1}, k_{2}$, and $k_{-2}$. In agreement with previous findings (Huet et al., 2006), the transition between ( and D is forbidden: SGs must dissociate from MTs and diffuse for a while within the actin cortex before they can attach at the PM. See Table 1 for a quantification of the rate constant values. Since the characteristic residency time at the cell periphery $(A+D, \sim 60 \mathrm{~s})$ is longer than the sum of the residency time in $A(\sim 15 \mathrm{~s})$ and in $D(\sim 15 \mathrm{~s})$, we conclude that SGs can undergo several local searches and successful membrane attachment periods before going back to $C$. $B$, Simulation of the photoconversion experiments. Shown is the comparison of the photoconversion experiment (control condition as in Fig. 5D, fitted with 2 exponentials; solid black line) and a simulation (gray) based on the model shown in $\boldsymbol{A}$ (for details, see Materials and Methods). The fast component reflects the equilibration between SGs attached to the PM and SGs diffusing in the actin-rich layer. The slow component represents the exit of SGs from the actin-rich layer.

pools, while the slow one (characteristic time, $\sim 130 \mathrm{~s}$, compared with $\sim 75$ s for the experimental curve) represents the exit of SGs from the actin-rich layer. Given the cell-to-cell dispersion of the experimental data and the fact that the simulations did not include any fitted parameter but rather used characteristics directly derived from independent experimental approaches, the simulations appear to follow reasonably well the experimental data, arguing for the validity of our compartment model.

The time spent by a SG in the actin-rich layer $\left[1 /\left(k_{2}+k_{-1}\right)\right]$ is about $10 \mathrm{~s}$, similar to the time spent in the immobile state. From our photoactivation data, we also obtained the overall time spent in the juxtamembrane region, a value close to $60 \mathrm{~s}$. Therefore, after microtubule-dependent delivery (long-range search), SGs perform an average of two successful local searches for an attachment site at the PM before leaving the juxtamembrane area.

The consequences of Myrip and MyoVa knockdown on SG dynamics at the cell cortex were analyzed using the same compartment model (Table 1). Myrip silencing induced a marked increase in $k_{-1}$ and $k_{-2}$ values and a decrease in $k_{2}$ values, indicating decreased retention in the actin-rich cell cortex, decreased transport toward attachment sites at the PM, and lowered stability of SG attachment. Lowering MyoVa levels induced a slight
Table 1. The dynamics of SGs quantified according to the model depicted in Figure 10

\begin{tabular}{lccc}
\hline & Control SiRNA & MyoVa SiRNA1 & \multicolumn{1}{c}{ Myrip SiRNA } \\
\hline Density $\left(\mathrm{SG} / \mu \mathrm{m}^{2}\right)$ & $0.44 \pm 0.038$ & $0.23 \pm 0.023^{* *}$ & $0.35 \pm 0.032$ \\
Docked $(\%)$ & $50.7 \pm 3.1$ & $35.6 \pm 3.7^{* *}$ & $24.4 \pm 2.4^{* * *}$ \\
$\mathrm{D}\left(\mathrm{SG} / \mu \mathrm{m}^{2}\right)$ & $0.22 \pm 0.025$ & $0.08 \pm 0.013^{* * *}$ & $0.08 \pm 0.011^{* *}$ \\
$\mathrm{~A}\left(\mathrm{SG} / \mu \mathrm{m}^{2}\right)$ & $0.22 \pm 0.025$ & $0.15 \pm 0.016$ & $0.26 \pm 0.028$ \\
$k_{2}\left(\mathrm{~s}^{-1}\right)$ & $0.082 \pm 0.012$ & $0.045 \pm 0.012^{*}$ & $0.040 \pm 0.006^{*}$ \\
$k_{-2}\left(\mathrm{~s}^{-1}\right)$ & $0.056 \pm 0.004$ & $0.064 \pm 0.01$ & $0.088 \pm 0.009^{* * *}$ \\
$k_{-1}\left(\mathrm{~s}^{-1}\right)$ & $0.016 \pm 0.002$ & $0.022 \pm 0.002^{* *}$ & $0.036 \pm 0.005^{* *}$ \\
$k_{1}\left(\mathrm{SG} / \mu \mathrm{m}^{2} / \mathrm{s}\right)$ & $0.0033 \pm 0.001$ & $0.0027 \pm 0.0002$ & $0.0087 \pm 0.001^{* *}$ \\
$N(\mathrm{cells})$ & 22 & 12 & 10 \\
\hline
\end{tabular}

SG density refers to the number of SGs detected by TIRFM. The density of SGs attached to the PM (D) or diffusing in the actin-rich layer $(A)$ were derived from $S G$ density and from the percentage of time spent in the stalled (immobile for more than $6-8.5 \mathrm{~s}$; see Materials and Methods) or in the diffusive state (Figure 8). The undocking rate constant $k_{-2}$ was derived from the exponential fit to the survival curves shown in Figure 7 (slow component of the curves). The rates of $S G$ appearance $\left(k_{1}\right)$ and disappearance $\left(k_{-1} A\right)$ from the TIRF area were measured as depicted in Figure 5. Since $A$ is known, we could obtain $k_{-1}$. Finally, at the steady state, $k_{2} A=k_{-2} D$; we thus obtained a value for $k_{2}$, the rate constant of $S G$ attachment to the PM. Data are expressed as the mean \pm SE of values obtained in the different cells. ${ }^{*} p<0.05 ;{ }^{* *} p<0.01 ;{ }^{* * *} p<0.001$.

increase in $k_{-1}$ and a decrease in $k_{2}$, but no significant change in the rate of SG detachment from the PM. Therefore, we conclude that the effects of Myrip can be partly accounted for by the recruitment of MyoVa and that Myrip also exerts a MyoVaindependent role in SG attachment to the PM. In addition, the effect of Myrip and MyoVa on $k_{2}$ suggest that the motor activity of MyoVa promotes the delivery of SGs at the PM, and therefore that MyoVa does not merely act as a tether.

\section{Myrip and MyoVa mediate the retention of SGs at the cell periphery}

Our observations demonstrate that Myrip and MyoVa mediate the retention of SGs at the cell periphery. Three mechanisms are likely involved. First, Myrip mediates the association of SGs with the actin-rich layer. This conclusion is supported by the increase in the rate constant $k_{-1}$ and the increased mobility of the SGs that were diffusing within the actin-rich layer observed in Myrip knockdown cells. Both MyoVa (Desnos et al., 2007b) and Myrip may mediate the association of SGs with actin filaments. The C-terminal region of Myrip, which directly interacts with F-actin (Fukuda and Kuroda, 2002; Desnos et al., 2003), contributes to the restriction in SG mobility during diffusive periods (see Results) (Fig. 9), suggesting that Myrip directly tethers SGs to actin.

The second mechanism that contributes to SG retention at the cell periphery is suggested by the effect of Myrip on SG motion along microtubules. SGs move faster along MTs in the absence of Myrip (present study) or MyoVa (Desnos et al., 2007b), suggesting that powering SG motion along microtubules is made more difficult in the actin-rich environment by the interaction of Myrip or MyoVa with F-actin. This interaction may therefore promote the detachment of the newly arrived SGs from microtubules. This capture mechanism involving dissociation from microtubules and retention in the actin-rich layer is likely to have broad implications in the polarized distribution of various organelles, such as melanosomes, calcium stores, or recycling endosomes (Desnos et al., 2007a; Wagner et al., 2011).

The third mechanism underlying SG retention in the subplasmalemmal region is the attachment of SGs to the PM. The probability of SG attachment to the PM (the A-to-D transition in Figure 10) is decreased in both Myrip and MyoVa knockdown cells, indicating that the two proteins promote SG delivery to release sites. This effect is probably due to the motor activity of MyoVa directed toward the PM, where F-actin barbed ends are enriched. In addition, we observed a specific effect of Myrip de- 
pletion on the stability of SG immobilization at the PM, indicating that Myrip, but not MyoVa, is a component of the attachment machinery. This effect is mediated by the C-terminal region of Myrip since overexpression of Myrip- $\Delta \mathrm{C}$ but not of full-length Myrip reduced the stability of SG immobilization.

Mizuno et al. (2011) previously characterized the effect of Myrip on the trafficking and exocytosis of SGs in insulinsecreting cells. In agreement with previous studies (Waselle et al., 2003; Ivarsson et al., 2005) and this report, they found that Myrip silencing reduced insulin secretion and SG recruitment at the cell periphery. Based on the effect of Myrip overexpression on the accumulation of SGs in the actin-rich layer, they suggested that Myrip only acts by tethering SGs to the actin-rich layer, via MyoVa. However, this observation supports the conclusion that Myrip tethers SGs to actin filaments (Desnos et al., 2003; Kuroda and Fukuda, 2005) (present study), but does not contradict the proposed additional role of Myrip in SG docking at the PM.

\section{A direct role for Myrip in SG attachment to the PM}

The fact that Myrip silencing and Myrip- $\Delta \mathrm{C}$, but not MyoVa, modify the duration of immobilization events indicates that the C-terminal region of Myrip is involved in the attachment of SGs to the PM. This region of Myrip interacts with components of the exocyst (Goehring et al., 2007), a multimeric protein complex involved in tethering reactions between membrane compartments. The exocyst targets several transport vesicles to the PM (Lipschutz and Mostov, 2002; He and Guo, 2009), and preliminary evidence supports its role in SG docking (Tsuboi et al., 2005). The reported effect of the C-terminal region of Myrip on SG secretion and melanosome distribution (Waselle et al., 2003; Imai et al., 2004; Ramalho et al., 2009) may therefore be related to the Myrip/exocyst interaction. Future studies will have to test the possibility that the exocyst complex mediates the effect of Myrip on SG docking. The SNARE proteins SNAP25 and syntaxin-1, as well as the syntaxin-1 partner Munc18 and the vesicular protein synaptotagmin-1, have also been implicated in SG docking at the PM (Toonen et al., 2006; de Wit et al., 2009). A key issue is to determine the relationship among Myrip, the exocyst, and this SNARE-based docking complex. One possibility is that Myrip mediates the first interaction with the PM, in concert with the large exocyst complex, and that SNAREs and synaptotagmins then assemble to mediate short-range interactions between SGs and the PM and prime SGs for exocytosis. The fact that previously immobilized SGs undergo a $20 \mathrm{~nm}$ step toward the PM (Karatekin et al., 2008) or a small lateral movement (Degtyar et al., 2007) shortly before fusion supports this possibility and suggests that different-sized tethering complexes are sequentially formed during the secretory process (Karatekin et al., 2008).

\section{References}

Chudakov DM, Lukyanov S, Lukyanov KA (2007) Tracking intracellular protein movements using photoswitchable fluorescent proteins PS-CFP2 and Dendra2. Nat Protoc 2:2024-2032.

Darchen F, Desnos C (2012) Masterclass with Rab3 and Rab27: orchestrating regulated secretion. In: Rab GTPases and membrane trafficking (Guangpu Li, Nava Segev, eds). Oak Park, IL: Bentham Science.

Degtyar VE, Allersma MW, Axelrod D, Holz RW (2007) Increased motion and travel, rather than stable docking, characterize the last moments before secretory granule fusion. Proc Natl Acad Sci U S A 104:15929-15934.

Desnos C, Schonn JS, Huet S, Tran VS, El-Amraoui A, Raposo G, Fanget I, Chapuis C, Menasche G, de Saint Basile G, Petit C, Cribier S, Henry JP, Darchen F (2003) Rab27A and its effector MyRIP link secretory granules to F-actin and control their motion towards release sites. J Cell Biol 163:559-570.
Desnos C, Huet S, Darchen F (2007a) “Should I stay or should I go?”: myosin V function in organelle trafficking. Biol Cell 99:411-423.

Desnos C, Huet S, Fanget I, Chapuis C, Bottiger C, Racine V, Sibarita JB, Henry JP, Darchen F (2007b) Myosin va mediates docking of secretory granules at the plasma membrane. J Neurosci 27:10636-10645.

de Wit H, Walter AM, Milosevic I, Gulyas-Kovacs A, Riedel D, Sorensen JB, Verhage M (2009) Synaptotagmin-1 docks secretory vesicles to syntaxin-1/SNAP-25 acceptor complexes. Cell 138:935-946.

El-Amraoui A, Schonn JS, Kussel-Andermann P, Blanchard S, Desnos C, Henry JP, Wolfrum U, Darchen F, Petit C (2002) MyRIP, a novel Rab effector, enables myosin VIIa recruitment to retinal melanosomes. EMBO Rep 3:463-470.

Espreafico EM, Cheney RE, Matteoli M, Nascimento AA, De Camilli PV, Larson RE, Mooseker MS (1992) Primary structure and cellular localization of chicken brain myosin-V (p190), an unconventional myosin with calmodulin light chains. J Cell Biol 119:1541-1557.

Evers BM, Townsend CM Jr, Upp JR, Allen E, Hurlbut SC, Kim SW, Rajaraman S, Singh P, Reubi JC, Thompson JC (1991) Establishment and characterization of a human carcinoid in nude mice and effect of various agents on tumor growth. Gastroenterology 101:303-311.

Fukuda M, Kuroda TS (2002) Slac2-c (synaptotagmin-like protein homologue lacking C2 domains-c), a novel linker protein that interacts with Rab27, myosin Va/VIIa, and actin. J Biol Chem 277:43096-43103.

Goehring AS, Pedroja BS, Hinke SA, Langeberg LK, Scott JD (2007) MyRIP anchors protein kinase A to the exocyst complex. J Biol Chem 282:33155-33167.

He B, Guo W (2009) The exocyst complex in polarized exocytosis. Curr Opin Cell Biol 21:537-542.

Huet S, Karatekin E, Tran VS, Fanget I, Cribier S, Henry JP (2006) Analysis of transient behavior in complex trajectories: application to secretory vesicle dynamics. Biophys J 91:3542-3559.

Imai A, Yoshie S, Nashida T, Shimomura H, Fukuda M (2004) The small GTPase Rab27B regulates amylase release from rat parotid acinar cells. J Cell Sci 117:1945-1953.

Ivarsson R, Jing X, Waselle L, Regazzi R, Renstrom E (2005) Myosin 5a controls insulin granule recruitment during late-phase secretion. Traffic 6:1027-1035.

Johns LM, Levitan ES, Shelden EA, Holz RW, Axelrod D (2001) Restriction of secretory granule motion near the plasma membrane of chromaffin cells. J Cell Biol 153:177-190.

Karatekin E, Tran VS, Huet S, Fanget I, Cribier S, Henry JP (2008) A 20-nm step toward the cell membrane preceding exocytosis may correspond to docking of tethered granules. Biophys J 94:2891-2905.

Kim M, Javed NH, Yu JG, Christofi F, Cooke HJ (2001) Mechanical stimulation activates Galphaq signaling pathways and 5-hydroxytryptamine release from human carcinoid BON cells. J Clin Invest 108:1051-1059.

Klomp AE, Teofilo K, Legacki E, Williams DS (2007) Analysis of the linkage of MYRIP and MYO7A to melanosomes by RAB27A in retinal pigment epithelial cells. Cell Motil Cytoskeleton 64:474-487.

Kuroda TS, Fukuda M (2005) Functional analysis of Slac2-c/MyRIP as a linker protein between melanosomes and myosin VIIa. J Biol Chem 280:28015-28022.

Lindsay AJ, McCaffrey MW (2011) Myosin Va is required for P body but not stress granule formation. J Biol Chem 286:11519-11528.

Lipschutz JH, Mostov KE (2002) Exocytosis: the many masters of the exocyst. Curr Biol 12:R212-R214.

Lopes VS, Ramalho JS, Owen DM, Karl MO, Strauss O, Futter CE, Seabra MC (2007) The ternary Rab27a-Myrip-Myosin VIIa complex regulates melanosome motility in the retinal pigment epithelium. Traffic 8:486-499.

Malacombe M, Bader MF, Gasman S (2006) Exocytosis in neuroendocrine cells: new tasks for actin. Biochim Biophys Acta 1763:1175-1183.

Michael DJ, Xiong W, Geng X, Drain P, Chow RH (2007) Human insulin vesicle dynamics during pulsatile secretion. Diabetes 56:1277-1288.

Mizuno K, Ramalho JS, Izumi T (2011) Exophilin8 transiently clusters insulin granules at the actin-rich cell cortex prior to exocytosis. Mol Biol Cell 22:1716-1726.

Nofal S, Becherer U, Hof D, Matti U, Rettig J (2007) Primed vesicles can be distinguished from docked vesicles by analyzing their mobility. J Neurosci 27:1386-1395.

Oheim M, Stuhmer W (2000) Tracking chromaffin granules on their way through the actin cortex. Eur Biophys J 29:67-89.

Ostrowski M, Carmo NB, Krumeich S, Fanget I, Raposo G, Savina A, Moita 
CF, Schauer K, Hume AN, Freitas RP, Goud B, Benaroch P, Hacohen N, Fukuda M, Desnos C, Seabra MC, Darchen F, Amigorena S, Moita LF, Thery C (2010) Rab27a and Rab27b control different steps of the exosome secretion pathway. Nat Cell Biol 12:19-30.

Racine V, Hertzog A, Jouanneau J, Salamero J, Kervrann C, Sibarita JB (2006) Multiple-target tracking of 3D fluorescent objects based on simulated annealing. Third IEEE International Symposium on Biomedical Imaging. pp 1020-1023. doi:10.1109/ISBI.2006.1625094.

Ramalho JS, Lopes VS, Tarafder AK, Seabra MC, Hume AN (2009) Myrip uses distinct domains in the cellular activation of myosin VA and myosin VIIA in melanosome transport. Pigment Cell Melanoma Res 22:461-473.

Rose SD, Lejen T, Casaletti L, Larson RE, Pene TD, Trifaro JM (2003) Myosins II and V in chromaffin cells: myosin V is a chromaffin vesicle molecular motor involved in secretion. J Neurochem 85:287-298.

Rudolf R, Salm T, Rustom A, Gerdes HH (2001) Dynamics of immature secretory granules: role of cytoskeletal elements during transport, cortical restriction, and F-actin-dependent tethering. Mol Biol Cell 12:1353-1365.

Rudolf R, Kogel T, Kuznetsov SA, Salm T, Schlicker O, Hellwig A, Hammer JA III, Gerdes HH (2003) Myosin Va facilitates the distribution of secretory granules in the F-actin rich cortex of PC12 cells. J Cell Sci 116:1339-1348.

Rudolf R, Bittins CM, Gerdes HH (2011) The role of myosin V in exocytosis and synaptic plasticity. J Neurochem 116:177-191.

Seabra MC, Coudrier E (2004) Rab GTPases and myosin motors in organelle motility. Traffic 5:393-399.
Siksou L, Rostaing P, Lechaire JP, Boudier T, Ohtsuka T, Fejtova A, Kao HT, Greengard P, Gundelfinger ED, Triller A, Marty S (2007) Threedimensional architecture of presynaptic terminal cytomatrix. J Neurosci 27:6868-6877.

Toonen RF, Kochubey O, de Wit H, Gulyas-Kovacs A, Konijnenburg B, Sorensen JB, Klingauf J, Verhage M (2006) Dissecting docking and tethering of secretory vesicles at the target membrane. EMBO J 25:3725-3737.

Tsuboi T, Ravier MA, Xie H, Ewart MA, Gould GW, Baldwin SA, Rutter GA (2005) Mammalian exocyst complex is required for the docking step of insulin vesicle exocytosis. J Biol Chem 280:25565-25570.

Varadi A, Tsuboi T, Rutter GA (2005) Myosin Va transports dense core secretory vesicles in pancreatic MIN6 beta-cells. Mol Biol Cell 16:2670-2680.

Verhage M, Sorensen JB (2008) Vesicle docking in regulated exocytosis. Traffic 9:1414-1424.

Wagner W, Brenowitz SD, Hammer JA, 3rd (2011) Myosin-Va transports the endoplasmic reticulum into the dendritic spines of Purkinje neurons. Nat Cell Biol 13:40-48.

Waselle L, Coppola T, Fukuda M, Iezzi M, El-Amraoui A, Petit C, Regazzi R (2003) Involvement of the Rab27 binding protein Slac2c/MyRIP in insulin exocytosis. Mol Biol Cell 14:4103-4113.

Yizhar O, Ashery U (2008) Modulating vesicle priming reveals that vesicle immobilization is necessary but not sufficient for fusion-competence. PLoS One 3:e2694. 